\title{
Parametric analysis of collapse load factor of planar frames
}

\author{
Erfan Maleki ${ }^{1}$ - Kazem Reza Kashyzadeh ${ }^{2}$
}

(c) Springer Nature Switzerland AG 2019

\begin{abstract}
The plastic analysis and determining the collapse load factor (CLF) of frames have received much attention in all kinematical methods applied for designing these frames. CLF in frames depends on many effective parameters such as length of the bay, the height of story, loads, and plastic moments. As the number of these parameters increases, the analysis becomes more complex and time-consuming. In this regard, artificial neural networks (ANNs) have been found many applications in prediction and optimization of ill-defined problems. In the present paper, two planar frames were considered and analyzed as examples for modeling via ANN. In order to develop the ANNs, results of thirty samples were gathered from every two examples. Parameters including the length of the bay, the height of story, loads, and plastic moments were regarded as inputs and the CLF was considered as the output of the networks. After developing the networks, the model function was generated and the effects of the input parameters were investigated on the CLF of frames. The results showed tuning networks are finely and adjusting their parameters would lead to very good and acceptable results for the design of frames.
\end{abstract}

Keywords Planar frame · Collapse load factor · Plastic analysis · Artificial neural network

\section{Introduction}

One of the most well-known techniques for calculating stability and design of frames is combining elementary mechanisms based on the analytical methods [1-3]. Similar to various methods provided to solve different kinds of engineering problems, this method also has limitations that prevent its use in all practical issues. The presence of rigid joints is one of the main problems in the plastic analysis of the frames that have been solved using linear programming by Charnes and Greenberg [4]. Moreover, plastic analysis and design of frames have some limitations $[5,6]$. Recently, some researchers have analyzed the behavior of the frames under different conditions [7-11].

Nguyen et al. presented nonlinear inelastic response history analysis of steel frame structures using plastic zone method [12]. Nguyen and Kim have performed nonlinear elastic dynamic analysis of space steel frames with semi-rigid connections $[13,14]$. Also, distributed plasticity approach has been used to perform a time-history analysis of steel frames $[15,16]$. In another study, the second-order spread of plasticity approach has been applied for nonlinear analysis of the space semi-rigid steel frames under time-history conditions [17]. Recently, the plastic zone method has been utilized to perform such an analysis [18]. Furthermore, the effects of various base restraints have been investigated on the nonlinear inelastic static and seismic responses of steel frames [19]. Greco et al. used a genetic algorithm to predict the seismic collapse of frame structures [20]. Moreover, a robust predictive model was presented by Aminian et al. based on the shear of steel frame structures using hybrid genetic programming [21]. Also, the neural network approach has been used to analyze the frames of conventional buildings [22]. Toward the developments in this field, a new algorithm has been presented for the automatic evaluation of plastic collapse

Erfan Maleki, maleki_erfan@kish.sharif.edu | ${ }^{1}$ Mechanical Engineering Department, Sharif University of Technology-International Campus, Kish Island, Iran. ${ }^{2}$ Mechanical Characteristics Lab, Center for Laboratory Services, Sharif University of Technology, Tehran, Iran.

SN Applied Sciences (2019) 1:514 | https://doi.org/10.1007/s42452-019-0532-2 
conditions for planar frames with vertical irregularities [23]. Besides, an improved ant colony optimization algorithm was introduced for the analysis of frame structures [24].

Among all studies conducted in this regard, there are two main methods for the design and analysis of frames. The first method is the finite element method (FEM) that is applied to compute the stiffness matrix of individual elements and assemble them into the global matrix of stiffness. Afterward, sets of related equations are solved to achieve the system response. Realizing the history of loadings is always necessary for the FEM method to analyze incrementally until the failure of the structure, although it is a time-consuming process $[5,6]$.

The second method includes algebraic methods that fall into a class of methods directly. In these techniques, the direct computation of the stiffness matrix is not essential and the system is assumed to be in the inception of failure. To combine elementary mechanisms, they are initially calculated by applying Gaussian elimination on a specific matrix. Next, they are combined to obtain a final collapse mechanism whose load factor is lower than all possible combinations of elementary mechanisms. This process presents the failure mechanism of the structure. In this method, the analysis of complete loading history is not needed and just the final collapse mechanism and the associated CLP value are evaluated.

Also, this method has a major difficulty that prevents its usage in a wide range of analyses. For example, in a two-dimensional frame, increasing the number of bays and stories results in the increase in the number of mechanisms are regarded in the combination process and makes the solution more complex [25]. Hence, obtaining a certain combination of elementary mechanisms that lead to highly accurate responses (considering the minimization of $(\mathrm{LF}$ ) is very costly and time-consuming. Considering the mentioned difficulties, in recent years, many attempts have been made on the identification and development of faster techniques to determine the CLP with high accuracy. Artificial intelligence (Al) systems can be used as an alternative approach in these cases. Al systems such as artificial neural networks (ANNs) have been used in many optimization and prediction problems. The most important advantage of these methods is their ability to solve complex problems and convergence of responses independent of the research scope. The main reason for this phenomenon is that these networks are easily adaptable to any topic. Hence, they are applied to optimize engineering problems in various industries. Cao et al. [26], Aydin et al. [27], Yahya et al. [28], Jiang et al. [29], and Jahanshahi et al. [30] have used ANNs for the analysis of accrued failures and damages in engineering structures and frames (2D and 3D).

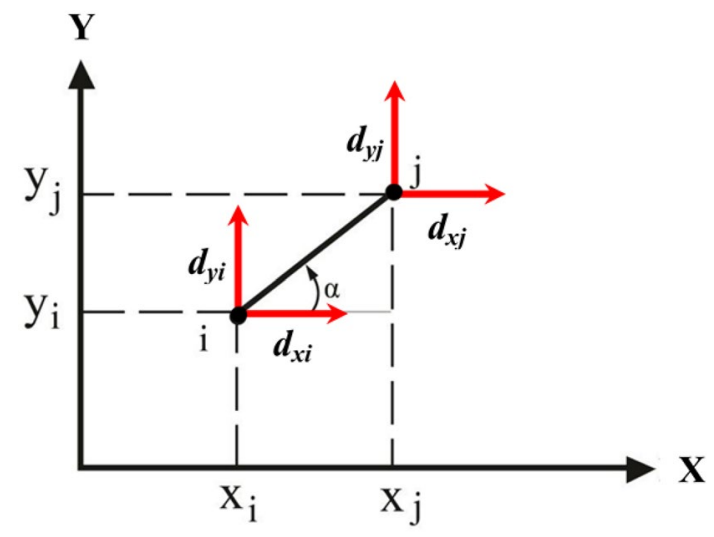

Fig. 1 End displacements of a pin-jointed rigid bar

The main aim of the present research is to develop a new approach based on ANNs to investigate the influences of effective parameters of planar frames on the CLF. To achieve this purpose, two planar frames were considered and analyzed as examples for the modeling process. A group of 30 samples was gathered from each of these two examples in order to build the ANN structure. Next, the developed network was tuned finely and their parameters were adjusted carefully.

\section{A brief description of the formation of elementary mechanisms}

Firstly, independent mechanisms were calculated for an assembly of pin-jointed rigid bars (Fig. 1). Here, the elongation of every bar is calculated via displacements of its two ends as follows:

$e=\left(d_{x j}-d_{x i}\right) \cos \alpha+\left(d_{y j}-d_{y i}\right) \sin \alpha$

where $e$ is the elongation and $d_{x i}$ and $d_{y i}$ are the end displacements for the $i$-th member along $X$ and $Y$ axes, respectively. In addition, $d_{x j}$ and $d_{y j}$ are the corresponding displacements for the end of $j$. It can be rewritten as a matrix equation that involves all the members.

$\mathbf{e}=\mathbf{C d}$

where e represents the elongation vector, $\mathrm{C}$ is the coefficient matrix, and $d$ is the displacement vector relating to the joints. The elementary mechanism is based on the principle that in an ideal mechanism the bars do not have elongation $(\mathrm{Cd}=0)$. The assembly is not stable with all joints pinned (the structure is not considered as a truss). Therefore, the difference between the number of columns and the number of rows related to the matrix $C$ is equal to the number of independent mechanisms. By performing 
Gaussian elimination on $\mathrm{Cd}=0$, results can be assessed as follows:

$\left[\mathbf{I}: \mathbf{C}_{d}\right]\left\{\begin{array}{c}\mathbf{d}^{i} \\ \mathbf{d}^{d}\end{array}\right\}=0$

By substituting the parameters into the Eq. (3), $\mathbf{d}^{i}$ will be achieved in terms of $\mathbf{d}^{d}$ as:

$\mathbf{d}^{i}=-\mathbf{C}_{d} \mathbf{d}^{d}$

To simplify the computational approximation, dependent vectors can be constructed using superposition theorem (setting one of the dependent displacements to unity and the others to zero). According to the Deeks method [31], independent mechanisms can be refined by removing the excess hinges for obtaining a set of potential collapse mechanisms. This can be achieved by checking every independent mechanism for containing all set of active hinges of another independent mechanism. This method is repeated so that no changes will be made to the system [30].

\section{Calculation of the CLF}

In the current study, the CLF was calculated by utilizing the virtual work theorem in which the ratio of internal to external virtual works represents the value of collapse load factor $(\lambda)$ as follows:

$\lambda=\frac{M^{T}|r|}{P^{T} d}$

where $\mathrm{P}$ and $\mathrm{d}$ represent joint force and corresponding joint displacement within the same direction, respectively, and $M$ and $r$ denote the plastic moment and rotations at hinges, respectively. Moreover, other research achivements remark that:"As the joint mechanisms are neglected throughout the formation of independent mechanisms, it is necessary to search out the locations of hinges in the members. These locations are determined to minimize the internal virtual work. If a joint is restrained against rotation, hinges are formed in all the members connected to this joint. However, if the joint is not restrained against rotation, hinges are formed in $(n-1)$ members among $n$ members connected to that joint" [25].

\section{Artificial neural networks}

ANN is one of the most well-known soft computing efficient tools that has been utilized for modeling different engineering problems. The method has been inspired by the human's brain and biological neural systems [32-34]. ANNs as highly interconnected arrays of processing computational neurons have been widely used and proven to be flexible interpolation functions. These networks are able to adapt to fit any advanced info and have the capability of prediction and optimization $[35,36]$. The principles of ANN modeling concerning the performance and application of the biological and artificial neurons has been studied in various works [37-39]. The main advantage of an ANN over usual numerical analysis procedures, under the provision that the predicted results fall within acceptable tolerances, is that results can be produced so fast, requiring orders of magnitude less computational effort than the common procedures [40]. A single neuron computes the sum of the entered inputs, which are multiplied with a variant called the weight, adds a bias term, and drives the result through a transfer function to produce a target output. Generally, linear, tangent sigmoid (Tansig), and logarithmic sigmoid (Logsig) functions are used as the transfer functions. These transfer functions are mathematically represented as follows:

Linear : $\chi(x)=\operatorname{linear}(x)$

Tansig : $\phi(x)=\frac{2}{1+e^{-2 x}}-1$

Logsig : $\psi(x)=\frac{1}{1+e^{-x}}$

Structurally, each ANN consists of an input layer, hidden layer/layers, and an output layer [41]. The structure of an ANN model is determined by the number of its layers, a respective number of nodes in every layer, and the nature of the transfer function [42]. Figure 2 presents a neural network that is fed with $p$ as input parameter and $a$ as output parameter, with weight matrixes $w$, bias vectors $b$, linear combiner $u$, and transfer function $f$.

\subsection{Implementation of ANN}

In order to simulate a subjet by ANN, two main steps of network training and testing should be regarded. The main difference between these two steps is the used data sets; those used for testing are not used through the training step. Training step includes the process of calculating the weights and biases values with adaptation, learning from, and evaluating the training patterns. Sets of known input and output data are used to train the network. To achieve the optimal structure of ANN with the least errors, there is no generally accepted rule. However, one among the difficult steps in ANN modeling is selecting the optimum neural network structure via trial and error [43]. Generally, this procedure is carried out by training different networks with different structures and comparing them to gain acceptable ranges of errors. In this study, some 


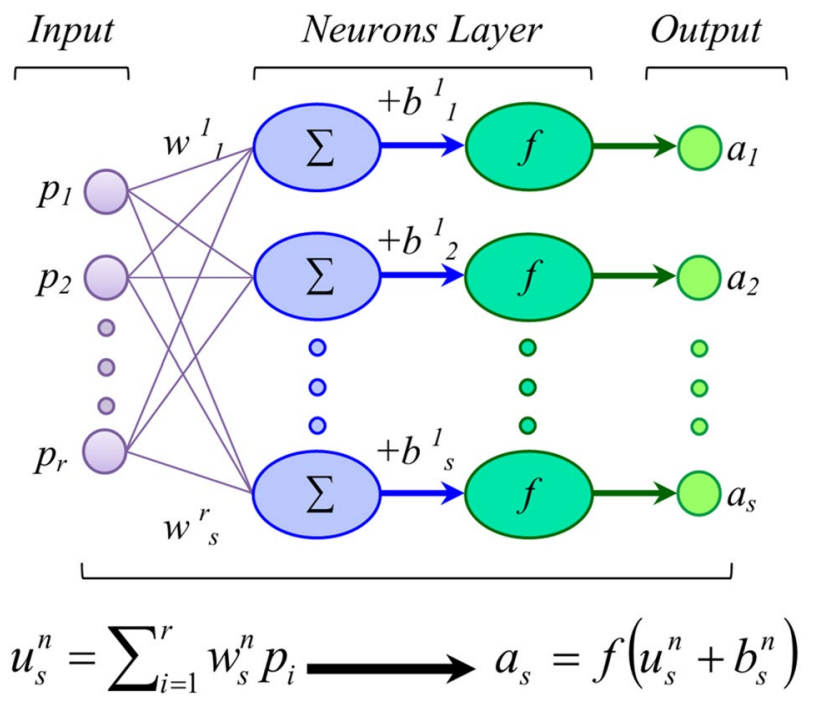

Fig. 2 One layer neural network that feed with $r$ inputs and $s$ outputs

well-known algorithms such as the feed-forward error back-propagation (BP) algorithm are employed to train the networks that are using a gradient descent technique to minimize the error for a particular training pattern. The accuracy of the developed model can increase along with the number of datasets [44]. In the present study, CLF of the planar frames was modeled using the ANN technique. Length of the bay, the height of story, loads, and plastic moments are regarded as inputs and the CLF considered as an output of the networks.

\subsection{Performance evaluation of ANN}

The efficiency of the developed ANN models during this research was assessed using several statistical criteria by comparing the experimental and predicted results of ANN. Four criteria of the coefficient of correlation $\left(R^{2}\right)$, root mean square error (RMSE), mean relative error (MRE), and mean absolute error (MAE) were used for this purpose (Eqs. 7-10):

$$
R^{2}=\frac{\sum_{i=1}^{n}\left(f_{E X P, i}-F_{E X P}\right)\left(f_{A N N, i}-F_{A N N}\right)}{\sqrt{\sum_{i=1}^{n}\left(\left(f_{E X P, i}-F_{E X P}\right)^{2}\left(f_{A N N, i}-F_{A N N}\right)^{2}\right)}}
$$

$$
R M S E=\sqrt{\frac{\sum_{i=1}^{n}\left(f_{E X P, i}-f_{A N N, i}\right)^{2}}{n}}
$$

MRE $=\frac{1}{n} \sum_{i=1}^{n} \frac{\left|f_{E X P, i}-f_{A N N, i}\right|}{f_{E X P, i}} \times 100$
$M A E=\frac{1}{n} \sum_{i=1}^{n}\left|f_{E X P, i}-f_{A N N, i}\right|$

where $n$ is the number of used sample for modeling step, $f_{E X P}$ is the experimental value, and $f_{A N N}$ is the value predicted by networks. Also, the values of $F_{E X P}$ and $F_{A N N}$ are calculated as follows:

$F_{E X P}=\frac{1}{n} \sum_{i=1}^{n} f_{E X P, i}$

$F_{A N N}=\frac{1}{n} \sum_{i=1}^{n} f_{A N N, i}$

\subsection{Generating model function}

After accomplishing successful training and achieving the optimum network structure, the values of weights and biases were obtained. The model function (in a four-layer network) can be determined as follows:

$a^{1}=f^{1}\left(w^{1} i+b^{1}\right)$

$a^{2}=f^{2}\left(w^{2} i^{1}+b^{2}\right)$

$a^{3}=f^{3}\left(w^{3} i^{2}+b^{3}\right)$

$a^{4}=f^{4}\left(w^{4} i^{3}+b^{4}\right)$

$M(m(1))=a^{4}=f^{4}\left(w^{4} f^{3}\left(w^{3} f^{2}\left(w^{2} f^{1}\left(w^{1} i+b^{1}\right)+b^{2}\right)+b^{3}\right)+b^{4}\right)$

where $a^{1}, a^{2}$, and $a^{3}$ are outputs of the first, second, and third layers, respectively, and $a^{4}$ is the fourth layer output, which is equal to the function $M(m(1))$. The function $M$ collects the values of all input parameters that are fed to the network. The desired outputs of CLF are depicted with $m(1)$.

\subsection{The used methodology of ANN}

The methodology of ANN is presented based on the error convergence using some well-known criteria including $R^{2}$, RMSE, MRE, and MAE. When the $R^{2}$ is close to 1 and both criteria of RMSE and MAE are close to zero, it can be stated that the developed network is efficient enough. Based on the results reported by Elangovan et al. [45] and Maleki et el. [37, 46, 47], attaining values of $R^{2}$ more than 0.99 are much acceptable for this criterion. The methodology used in the current study is shown in Fig. 3. 
Fig. 3 The used methodology consisting of network training, investigation of the results achieved from ANN and results evaluation

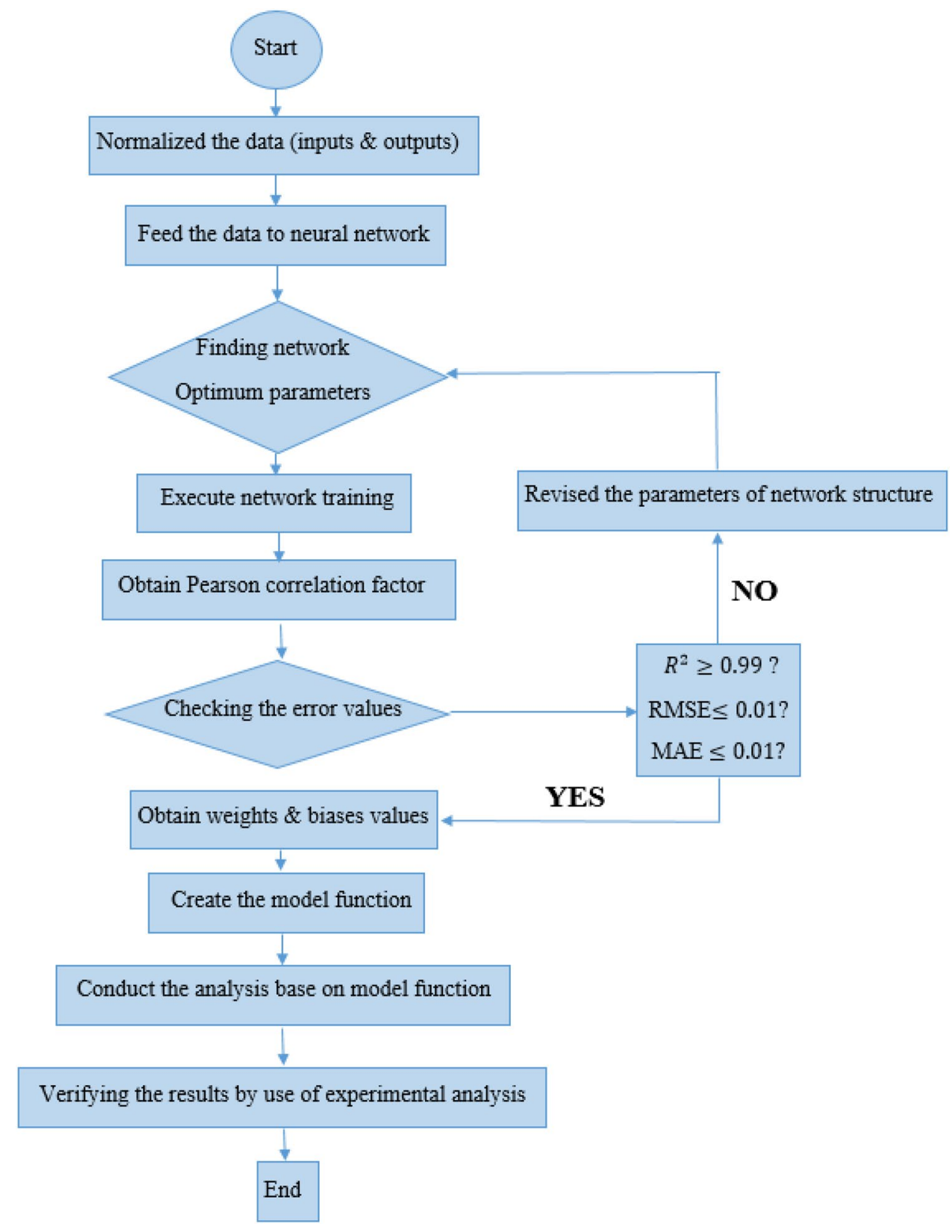

\section{Numerical results}

In this section, two numerical examples are presented (Fig. 4) to analyze the obtained results. The first example is a one-bay and one-story and the other is a two-bay and two-story frame under horizontal and vertical forces and plastic moments. The exact CLF was determined using the method of combination of elementary mechanisms. The related data of each example (Tables 1 and 2) were used to develop an ANN for the modeling process. A package of 30 samples is employed for training and testing the networks for every example. A total of 25 samples data (83\%) were employed as data sets for network training. The network testing is performed using 5 sample data (17\%), which were not used during training. The conceptual schematic structure of the used four layers ANN, using feed-forward BP algorithm and fully interconnected neurons for modeling of both examples, is illustrated in Fig. 5 .

Various networks were trained to achieve the optimal structures of both examples modeling for generating the correspondence model functions. Related information of 5 different trained networks with trial and error approach for modeling of CLF of both example are presented in Tables 3 and 4, respectively. After investigating the trained network, ANN models with structures of $6 \times 12 \times 14 \times 1$ and $8 \times 14 \times 14 \times 1$ that have the highest value of $R^{2}$ and the 
Fig. 4 Geometry, plastic moments and loadings of considering example frames. a One-bay and One-story frame for example 1 and $\mathbf{b}$ Twobay and Two-story frame for example 2

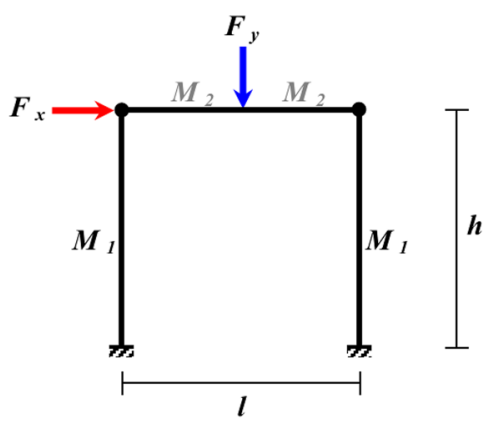

(a)

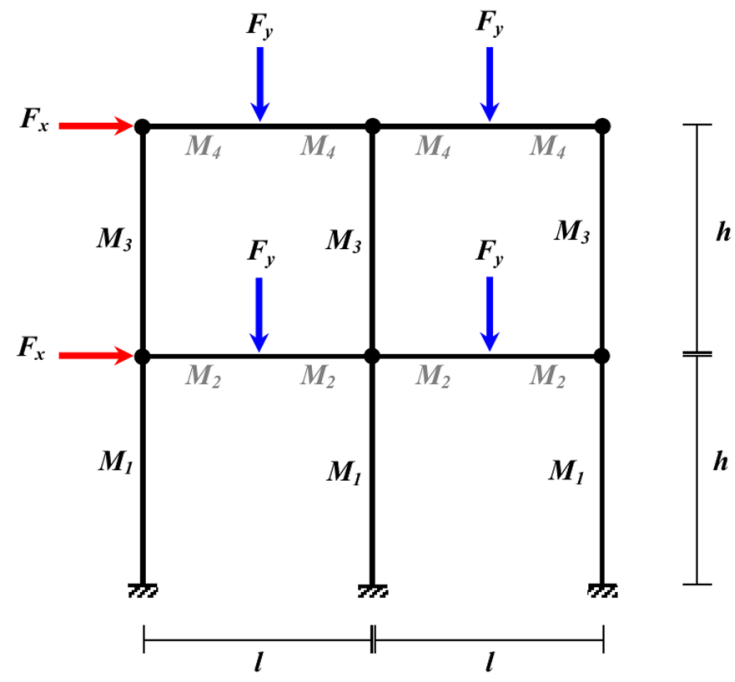

(b)
Table 1 Values of effective parameters for 30 sample frames of example 1

\begin{tabular}{|c|c|c|c|c|c|c|c|c|}
\hline Sample no. & I & $H$ & $M_{1}$ & $M_{2}$ & $F_{x}$ & $F_{y}$ & $\lambda$ & Sample Type \\
\hline 1 & 2 & 2 & 1 & 1 & 1 & 1 & 2.00000 & Training \\
\hline 2 & 2 & 3 & 1 & 1 & 1 & 2 & 1.20000 & \\
\hline 3 & 2 & 3.5 & 1.5 & 1.5 & 1.5 & 1.5 & 1.14286 & \\
\hline 4 & 2 & 3.75 & 1.75 & 1.75 & 1.25 & 2 & 1.49333 & \\
\hline 5 & 2 & 4 & 1.75 & 1 & 1 & 1.75 & 1.30435 & \\
\hline 6 & 2.25 & 2.25 & 1.25 & 1.25 & 1.25 & 1.25 & 1.77778 & \\
\hline 7 & 2.5 & 2.5 & 1.5 & 1.5 & 1.5 & 1.5 & 1.60000 & \\
\hline 8 & 2.5 & 4 & 1.25 & 1.25 & 1.25 & 1.75 & 1.00000 & \\
\hline 9 & 2.75 & 2.75 & 1.75 & 1.75 & 1.75 & 1.75 & 1.45455 & \\
\hline 10 & 3 & 3 & 2 & 2 & 2 & 2 & 1.33333 & \\
\hline 11 & 3 & 3.5 & 2 & 2 & 1 & 1 & 2.28571 & \\
\hline 12 & 3 & 4 & 2 & 1 & 1 & 2 & 1.14286 & \\
\hline 13 & 4 & 3.75 & 1.5 & 1.75 & 1.5 & 1.75 & 1.04110 & \\
\hline 14 & 4 & 3.75 & 1.5 & 1.5 & 1.75 & 1.75 & 0.89441 & \\
\hline 15 & 4.5 & 4 & 2 & 2 & 1 & 2 & 1.41176 & \\
\hline 16 & 5.25 & 2 & 1.75 & 1.25 & 1 & 2 & 0.95238 & \\
\hline 17 & 5.5 & 2.5 & 1.75 & 1.5 & 1.25 & 1.75 & 1.19685 & \\
\hline 18 & 5.5 & 3.5 & 2 & 1 & 1 & 2 & 0.72727 & \\
\hline 19 & 5.5 & 3.75 & 2 & 1 & 1.25 & 2 & 0.72727 & \\
\hline 20 & 5.75 & 4 & 1 & 1 & 1 & 2 & 0.61538 & \\
\hline 21 & 6 & 2 & 2 & 1 & 1 & 2 & 0.66667 & \\
\hline 22 & 6 & 2.75 & 1.5 & 1.25 & 1.25 & 2 & 0.83333 & \\
\hline 23 & 6 & 3 & 2 & 1 & 1.25 & 1.75 & 0.76190 & \\
\hline 24 & 6 & 3.5 & 1.5 & 1.25 & 1.75 & 2 & 0.65975 & \\
\hline 25 & 6 & 4 & 2 & 2 & 1.5 & 2 & 1.00000 & \\
\hline 26 & 4.5 & 4 & 2 & 1 & 2 & 1 & 0.75000 & Testing \\
\hline 27 & 4.75 & 3 & 1 & 1 & 1.25 & 1.5 & 0.82051 & \\
\hline 28 & 4.75 & 3.25 & 1.5 & 1 & 1 & 1.25 & 1.12585 & \\
\hline 29 & 5 & 2 & 2 & 2 & 1.5 & 1.5 & 1.77777 & \\
\hline 30 & 5 & 3 & 1.5 & 1.5 & 1.75 & 2 & 0.87805 & \\
\hline
\end{tabular}


Table 2 Values of effective parameters for 30 sample frames of example 2

\begin{tabular}{|c|c|c|c|c|c|c|c|c|c|c|}
\hline Sample no. & $L$ & $H$ & $M_{1}$ & $M_{2}$ & $M_{3}$ & $M_{4}$ & $F_{x}$ & $F_{y}$ & $\lambda$ & Sample Type \\
\hline 1 & 2 & 2 & 2.5 & 2 & 1 & 0.5 & 1 & 1 & 1.94444 & Training \\
\hline 2 & 2 & 2.5 & 2.5 & 2 & 1 & 0.5 & 1 & 1.25 & 1.55556 & \\
\hline 3 & 2 & 3 & 2.5 & 2 & 0.5 & 0.25 & 1 & 1.25 & 0.80000 & \\
\hline 4 & 2 & 3.5 & 2.5 & 2 & 1 & 0.75 & 1 & 1.25 & 1.34615 & \\
\hline 5 & 2 & 4 & 2 & 1.5 & 1 & 0.75 & 1 & 1.25 & 1.08333 & \\
\hline 6 & 2.5 & 2 & 2 & 1.5 & 0.75 & 0.5 & 1 & 1.25 & 1.28000 & \\
\hline 7 & 2.5 & 2.5 & 2 & 1.5 & 0.75 & 0.5 & 1.25 & 1 & 1.09474 & \\
\hline 8 & 2.5 & 3 & 2 & 1.5 & 0.75 & 0.5 & 1.25 & 1 & 0.94545 & \\
\hline 9 & 2.5 & 3.5 & 2.5 & 2 & 0.75 & 0.5 & 1.25 & 1 & 0.92800 & \\
\hline 10 & 2.5 & 4 & 2.5 & 2 & 0.75 & 0.5 & 1.25 & 1 & 0.82857 & \\
\hline 11 & 3 & 4 & 2.5 & 2 & 0.5 & 0.25 & 1.25 & 1.5 & 0.44444 & \\
\hline 12 & 3 & 4 & 2.5 & 2 & 0.5 & 0.25 & 1 & 2 & 0.33333 & \\
\hline 13 & 3 & 4 & 2.5 & 1 & 1 & 0.75 & 1 & 2 & 0.89583 & \\
\hline 14 & 3 & 4 & 2 & 1.75 & 0.75 & 0.25 & 1.5 & 2 & 0.33333 & \\
\hline 15 & 3 & 3 & 2 & 1.75 & 2 & 1 & 1.5 & 2 & 1.11538 & \\
\hline 16 & 4 & 2.5 & 2 & 1.25 & 2 & 1 & 1.5 & 1.75 & 1.14286 & \\
\hline 17 & 4 & 2.5 & 2 & 1.25 & 2 & 1.5 & 1.5 & 1.75 & 1.32184 & \\
\hline 18 & 4 & 4 & 2 & 1.5 & 2 & 1.5 & 2 & 1.5 & 0.75000 & \\
\hline 19 & 4 & 4 & 1.5 & 1 & 1.5 & 0.75 & 2 & 1.5 & 0.51852 & \\
\hline 20 & 4 & 3 & 1.5 & 1 & 1.5 & 1 & 2 & 1.5 & 0.71429 & \\
\hline 21 & 5 & 3 & 1.5 & 1.25 & 2 & 0.75 & 2 & 1.25 & 0.67647 & \\
\hline 22 & 5 & 2 & 2.5 & 2 & 1 & 0.75 & 1 & 1.25 & 0.80000 & \\
\hline 23 & 5 & 2 & 2.5 & 2 & 0.75 & 0.5 & 1 & 2 & 0.40000 & \\
\hline 24 & 5 & 3.5 & 2.5 & 1.75 & 0.75 & 0.5 & 1.75 & 1 & 0.61674 & \\
\hline 25 & 5 & 4 & 2.5 & 1.75 & 1.5 & 1 & 1.25 & 2 & 0.78571 & \\
\hline 26 & 3.5 & 3 & 2 & 1.75 & 2 & 1.75 & 1.5 & 1.5 & 1.33333 & Testing \\
\hline 27 & 3.5 & 3 & 1.5 & 1 & 1 & 0.75 & 1.5 & 1.75 & 0.72727 & \\
\hline 28 & 3.5 & 2 & 1.5 & 1 & 2 & 1.5 & 1.5 & 1.75 & 1.42149 & \\
\hline 29 & 3.5 & 2 & 1.5 & 1.25 & 1.5 & 1 & 1.5 & 2 & 1.06522 & \\
\hline 30 & 3.5 & 2.5 & 2 & 1.75 & 2 & 1 & 1.5 & 1.75 & 1.25994 & \\
\hline
\end{tabular}

least values of RMSE, MRE, and MAE were selected as an optimal structure to generate the model functions. Figures 6 and 7 present the comparative diagrams of predicted and experimental values for both training and testing samples of both examples, respectively. Figure 8 represents obtained relative error (RE) values of CLF for both training and testing samples of examples 1 and 2 .

Table 5 presents the data used for the evaluation of the ANN via mentioned statistical criteria for both training and testing data sets.

According to the obtained results, in network training and testing processes, it is observed that the values of $R^{2}$ are more than 0.999 and the values of RMSE, MRE, and MAE are close to 0 and acceptable for the predicting the output parameter (i.e., CLF) in both example 1 and 2. Therefore, it is concluded that networks were trained efficiently and adjusted carefully. In modeling of each example, some mutations are observed in errors of different samples. The reason for this phenomenon is the inability of the neural network trained by the error back-propagation algorithm to converge while simulating data in a wider range. In addition, the obtained values of statistical criteria for the testing process in the modeling of both examples were increased negligibly and the obtained error values in both models were less than 1\%, which is an acceptable value.

After evaluating the developed networks, the modeling functions of examples 1 and 2 are generated discretely. Afterward, to have parametric analysis on plastic behavior of the considered planar frames, we determined the effects of effective parameters including length of the bay, the height of story, loads, and plastic moments on CLF. The generated functions of examples 1 and 2 are described by Eqs. $14 \mathrm{a}$ and $14 \mathrm{~b}$, respectively:

$$
\begin{aligned}
& \lambda_{\text {ex. } 1}=f\left(h, l, M_{1}, M_{2}, F_{x}, F_{y}\right) \\
& \lambda_{\text {ex. }}=f\left(h, l, M_{1}, M_{2}, M_{3}, M_{4}, F_{x}, F_{y}\right)
\end{aligned}
$$




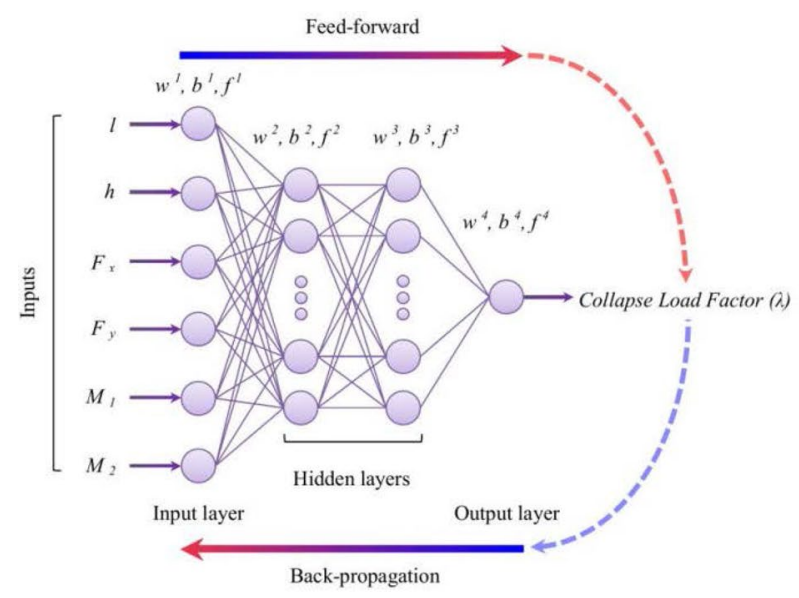

(a)

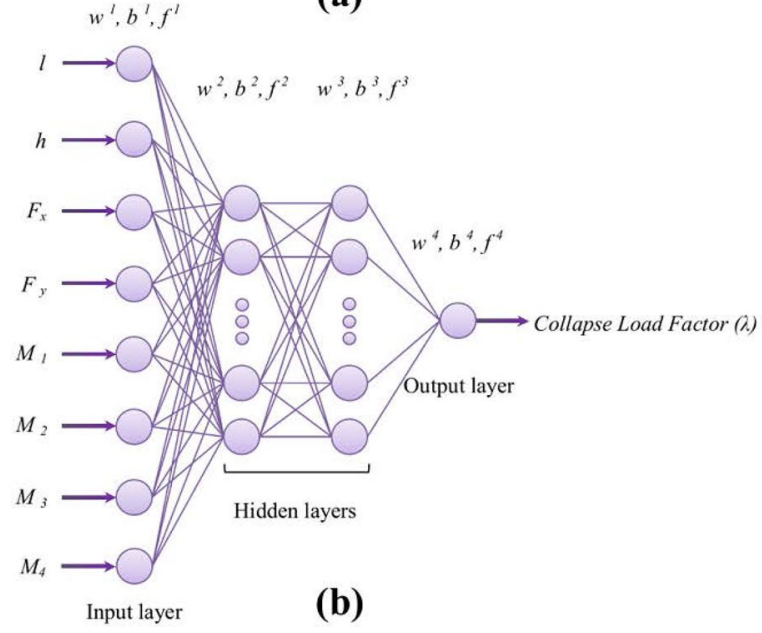

Fig. 5 Conceptual architecture of ANN according to the considered input and output parameters of the network using fee-forward and back-propagation algorithm related to a Example 1 and $\mathbf{b}$ Example 2

Figures 9 and 10 present the effects of input parameters on CLF obtained via generated model functions of the selected developed networks for examples 1 and 2 , respectively. Figure 9a-e present the effects of the whole parameters versus the value of CLF for the parameters related to the length of the bay, the height of story, loads and plastic moments, respectively.
According to the obtained results relevant to example 1 , to have greater CLF, if the value of $h$ is ascending, the value of I has to be descending; conversely, if the value of $I$ is ascending, the value of $h$ has to be descending. Also, if the applied loads (i.e., both $F_{x}$ and $F_{y}$ ) are increasing, the value of $I$ has to be reduced but if the value of parameter $F_{y}$ is descending the parameter $h$ has to rise to have more CLF. On the other hand, if the $F_{x}$ is ascending, the value of $h$ must be descending. About the plastic moments, it can be seen that to have greater CLF, by increasing the parameter $M_{1}$, the parameters $l, h$ and $F_{y}$ have to decrease whereas the other parameters of $M_{2}$ and $F_{x}$ have to enhance simultaneously. Moreover, about the effects of the applied loads, it can be observed that by a decrease in $F_{y}$, the value of the parameter $F_{x}$ has to be ascending.

In addition, the comparison of the influences of the some mentioned parameters in example 1 for several specific values is presented in "Appendix 1". As can be seen, the parameter of the plastic moment $\left(M_{1}\right)$ has interesting effects; to gain a higher CLF and greater $I$ and $h$, a turning point is made. Before that point, $M_{1}$ has to decrease and after that, the value of $M_{1}$ has to rise. This feature occurs in the plastic moment parameter $\left(M_{2}\right)$ of example 2, as well.

For the parametric analysis of influences of the mentioned parameters in example 2, the effects of some parameters are investigated (Fig. 10). For instance, it can be observed that by decreasing the parameter $I$, the parameters $h, F_{x}$ and $F_{y}$ must have a reduction as well. Overall, to have more $C L F$, the related values for the length of the bays, the height of the stories, and applied loads have to become as lower as possible; in other words, the CLF has reverse relation with $I, h$, and $F_{x}$ and $F_{y}$. Figure $10 \mathrm{~b}$ illustrates the effects of all applied moments on CLF, with which each of them having different specific influence. For example, it is seen that if the value of the parameter $M_{4}$ is raising, the other moments of $M_{1}, M_{2}$, and $M_{3}$ have to become lower to increase the CLF. Same as example 1, the comparison of the influences of the some mentioned parameters in example 2 for several specific values is presented in "Appendix 2".

Table 3 Related information of 5 different network training for modeling of CLF for example 1

\begin{tabular}{|c|c|c|c|c|c|c|c|c|}
\hline $\begin{array}{l}\text { ANN mod- } \\
\text { eling no. }\end{array}$ & Rate of training & Layers structure & $\begin{array}{l}\text { Hidden transfer } \\
\text { function }\end{array}$ & $\begin{array}{l}\text { Output transfer } \\
\text { function }\end{array}$ & $\mathrm{R}^{2}$ & RMSE & MRE (\%) & MAE \\
\hline 1 & 0.115 & $6 \times 8 \times 8 \times 1$ & Logsig & Linear & 0.9863 & 0.0048 & 0.1948 & 0.0044 \\
\hline 2 & 0.120 & $6 \times 8 \times 10 \times 1$ & Tansig & Tansig & 0.9888 & 0.0042 & 0.1840 & 0.0039 \\
\hline 3 & 0.120 & $6 \times 10 \times 12 \times 1$ & Logsig & Linear & 0.9911 & 0.0033 & 0.1777 & 0.0034 \\
\hline 4 & 0.130 & $6 \times 12 \times 12 \times 1$ & Tansig & Linear & 0.9955 & 0.0026 & 0.1506 & 0.0030 \\
\hline 5 & 0.135 & $6 \times 12 \times 14 \times 1$ & Logsig & Logsig & 0.9999 & 0.0023 & 0.1372 & 0.0026 \\
\hline
\end{tabular}


Table 4 Related information of 5 different network training for modeling of CLF for example 2

\begin{tabular}{|c|c|c|c|c|c|c|c|c|}
\hline $\begin{array}{l}\text { ANN mod- } \\
\text { eling no. }\end{array}$ & Rate of training & Layers structure & $\begin{array}{l}\text { Hidden transfer } \\
\text { function }\end{array}$ & $\begin{array}{l}\text { Output transfer } \\
\text { function }\end{array}$ & $\mathrm{R}^{2}$ & RMSE & MRE (\%) & MAE \\
\hline 1 & 0.120 & $8 \times 8 \times 10 \times 1$ & Tansig & Linear & 0.9831 & 0.0050 & 0.3919 & 0.0054 \\
\hline 2 & 0.125 & $8 \times 8 \times 12 \times 1$ & Tansig & Linear & 0.9876 & 0.0041 & 0.3487 & 0.0051 \\
\hline 3 & 0.125 & $8 \times 10 \times 12 \times 1$ & Logsig & Logsig & 0.9914 & 0.0034 & 0.3004 & 0.0046 \\
\hline 4 & 0.135 & $8 \times 12 \times 14 \times 1$ & Logsig & Tansig & 0.9983 & 0.0029 & 0.2765 & 0.0038 \\
\hline 5 & 0.140 & $8 \times 14 \times 14 \times 1$ & Logsig & Logsig & 0.9998 & 0.0025 & 0.2254 & 0.0031 \\
\hline
\end{tabular}

(a)

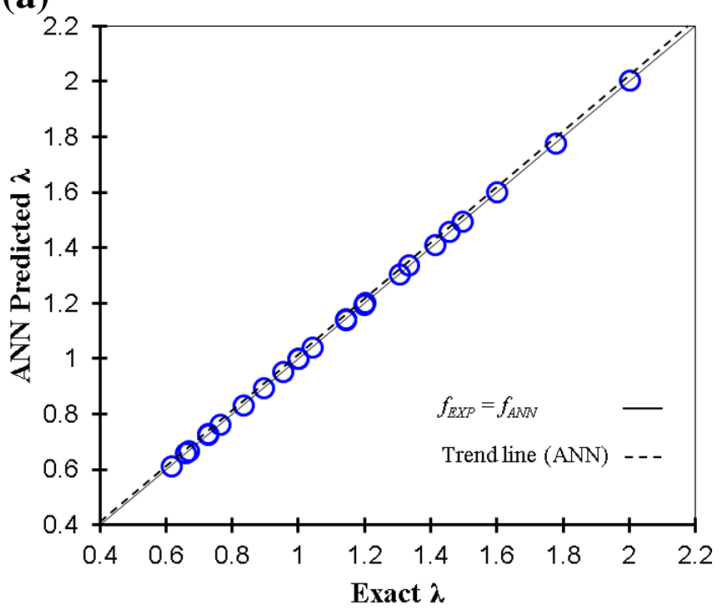

(b)

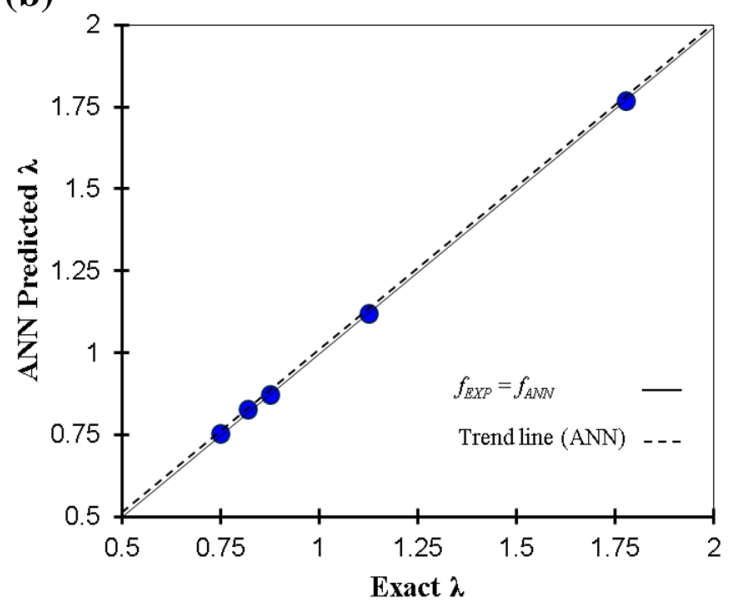

Fig. 6 Comparison of the predicted (ANN response) and experimental values for each $\mathbf{a}$ training and $\mathbf{b}$ testing samples of example 1

(a)

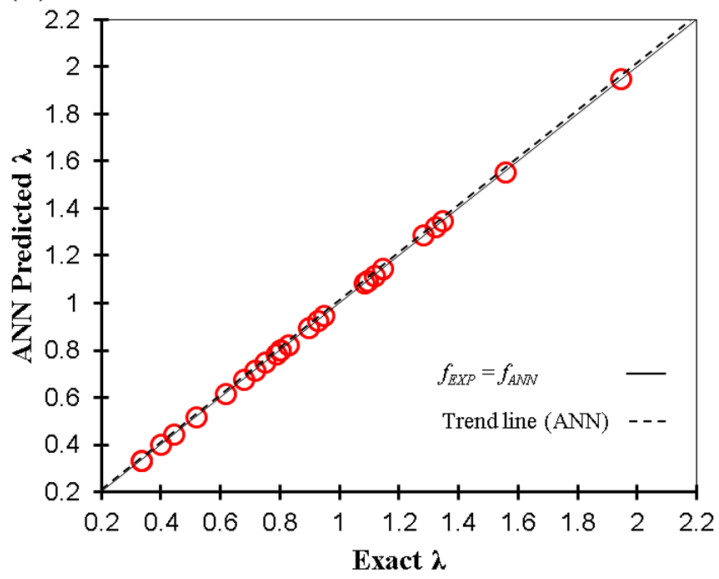

(b)

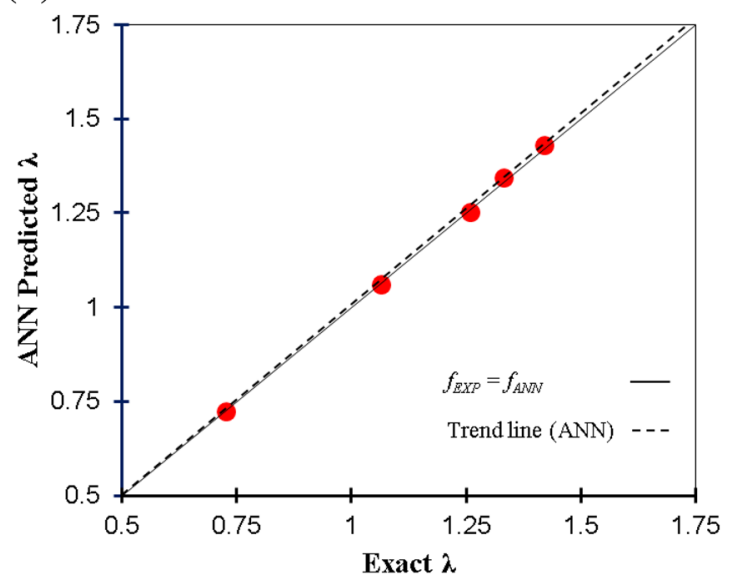

Fig. 7 Comparison of the predicted (ANN response) and experimental values for each $\mathbf{a}$ training and $\mathbf{b}$ testing samples of example 2

After parametric analysis, to analyze the sensitivity and assess the relative importance of the input variables of each example, an evaluation process was used based on the neural network-weight matrix and Garson equation, which is relation based on partitioning the connection weights [30]:

$$
I_{j}=\frac{\sum_{m=1}^{N_{h}}\left(\left(\frac{\left|w_{j m}^{i h}\right|}{\sum_{k=1}^{N_{i}}\left|w_{k m}^{i h}\right|}\right) \times\left|W_{m n}^{h o}\right|\right)}{\sum_{k=1}^{N_{i}}\left\{\sum_{m=1}^{N_{h}}\left(\frac{\left|w_{k m}^{i h}\right|}{\sum_{k=1}^{N_{i}}\left|w_{k m}^{\text {ih }}\right|}\right) \times\left|W_{m n}^{h o}\right|\right\}}
$$


(a)

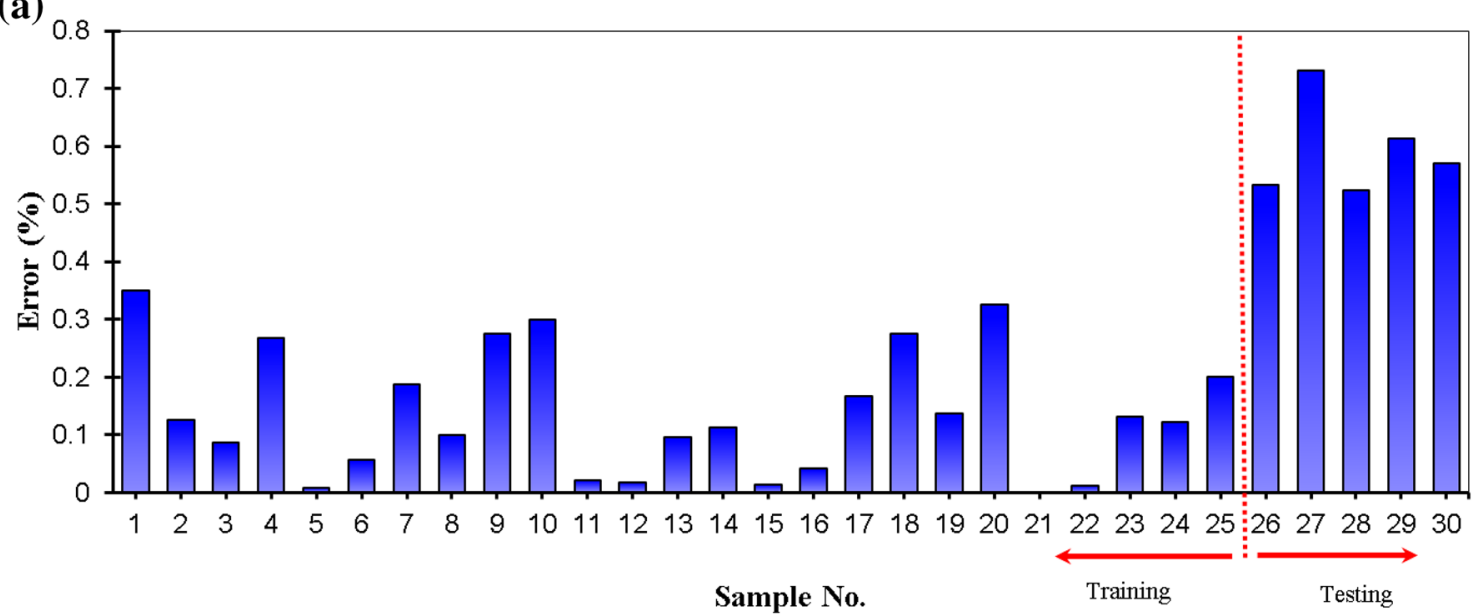

(b)

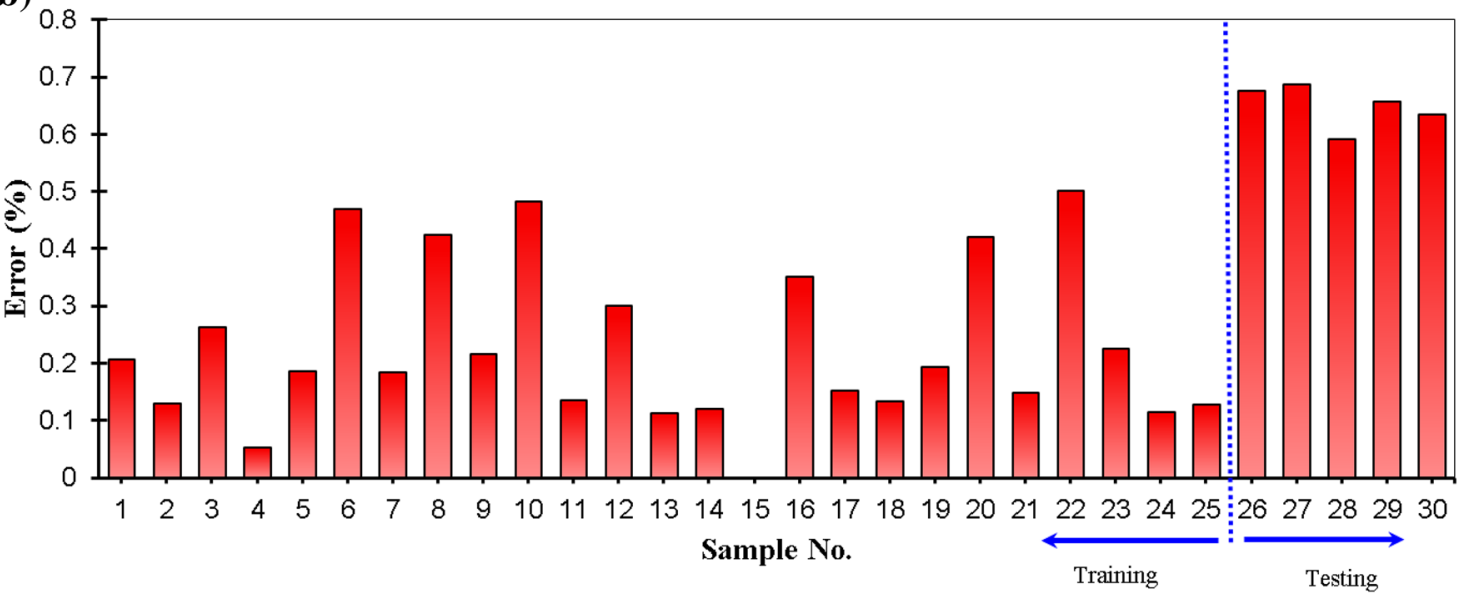

Fig. 8 Values of relative errors obtained for both training and testing data set relevant to $\mathbf{a}$ example 1 and $\mathbf{b}$ example 2

Table 5 Obtained values of $\mathrm{R}^{2}, \mathrm{RMSE}, \mathrm{MRE}$, and MAE for trained and tested network

\begin{tabular}{|c|c|c|c|c|c|c|c|c|}
\hline \multirow[t]{2}{*}{ Predicted CLF } & \multicolumn{4}{|c|}{ Network training } & \multicolumn{4}{|c|}{ Network testing } \\
\hline & $\mathrm{R}^{2}$ & RMSE & MRE (\%) & MAE & $\overline{R^{2}}$ & RMSE & MRE (\%) & MAE \\
\hline Example 1 & 0.9999 & 0.0023 & 0.1372 & 0.0026 & 0.9995 & 0.0068 & 0.5942 & 0.0064 \\
\hline Example 2 & 0.9998 & 0.0025 & 0.2254 & 0.0031 & 0.9993 & 0.0076 & 0.6491 & 0.0075 \\
\hline
\end{tabular}

where $l_{j}$ is the importance of $j$ th input variable related to the output variable, $N_{i}$ and $N_{h}$ are the numbers of inputs and hidden neurons, respectively, $W$ is the connection weight, and the superscripts $i, h$, and $o$ refer to input, hidden and output neurons, respectively.

The relative importance of the inputs is demonstrated in Fig. 11. It was found that all variables strongly affect the value of CLF in each considered frames. The ranking and the value of importance for each regarded inputs are shown in Table 6.

This study was conducted to investigate parametric plastic analysis of two simple planar frames via ANN. The obtained results showed that ANN could be used as an alternative and efficient tool to accomplish this analysis. In addition, the results can be useful in the design of frames. For future studies, this method can be employed for structures that are more complex and with a larger number of bays, stories, loads and plastic moments.

\section{Conclusion}

In the present study, the influences of effective parameters on the collapse of planar frames are investigated and modeled via ANN. In order to develop a network, the length of the bay, the height of story, loads and plastic moments 

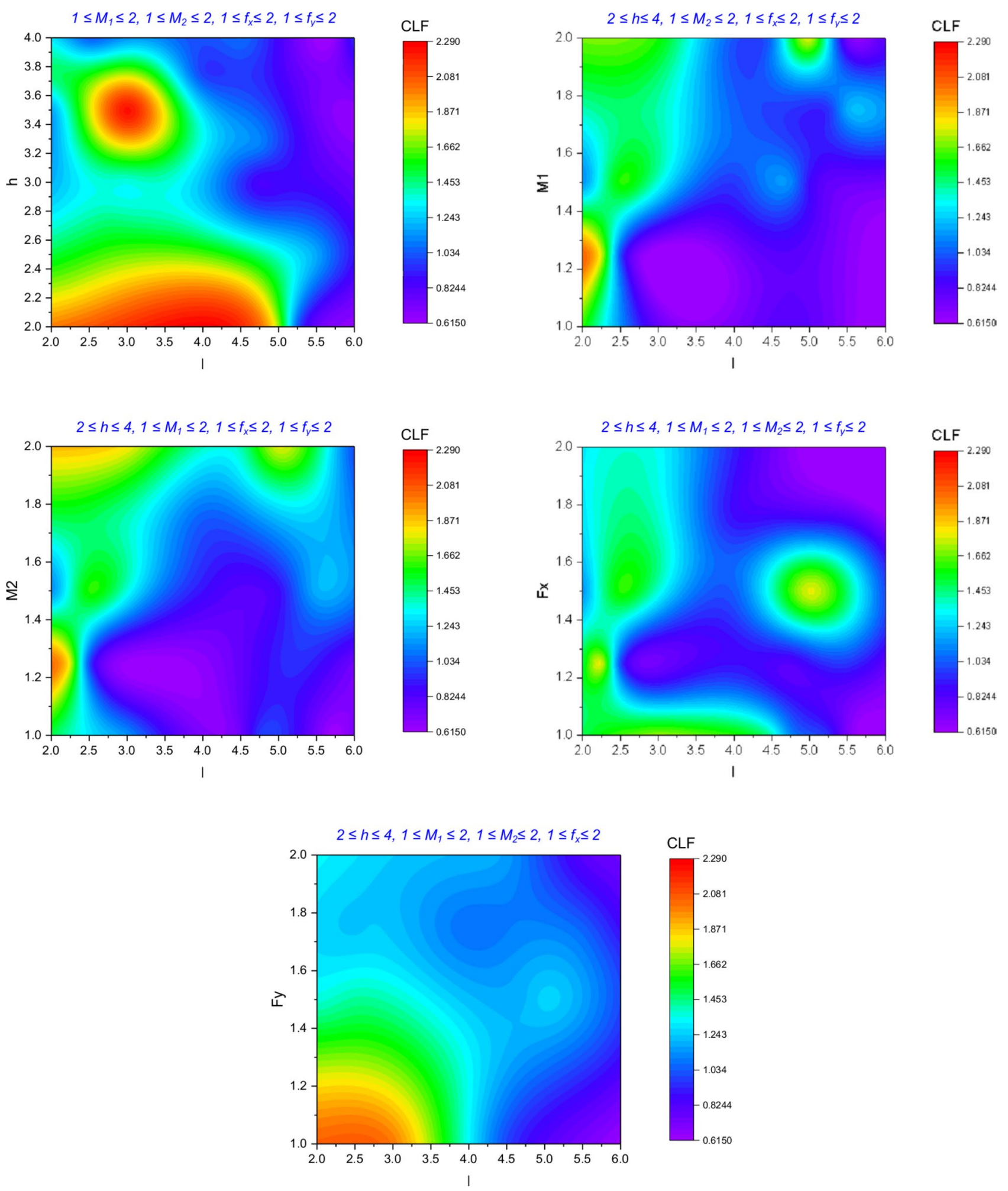

(a)

Fig. 9 Parametric analysis of input parameters including length of bay $l$, height of story $h$, and plastic moments $M$ influences on CLF obtained via generated model functions of the selected developed

network for example 1; effects of a length of bays, $\mathbf{b}$ height of stories, c horizontal force, $\mathbf{d}$ vertical force, and e plastic moments and the other effective parameters all together on CLF 

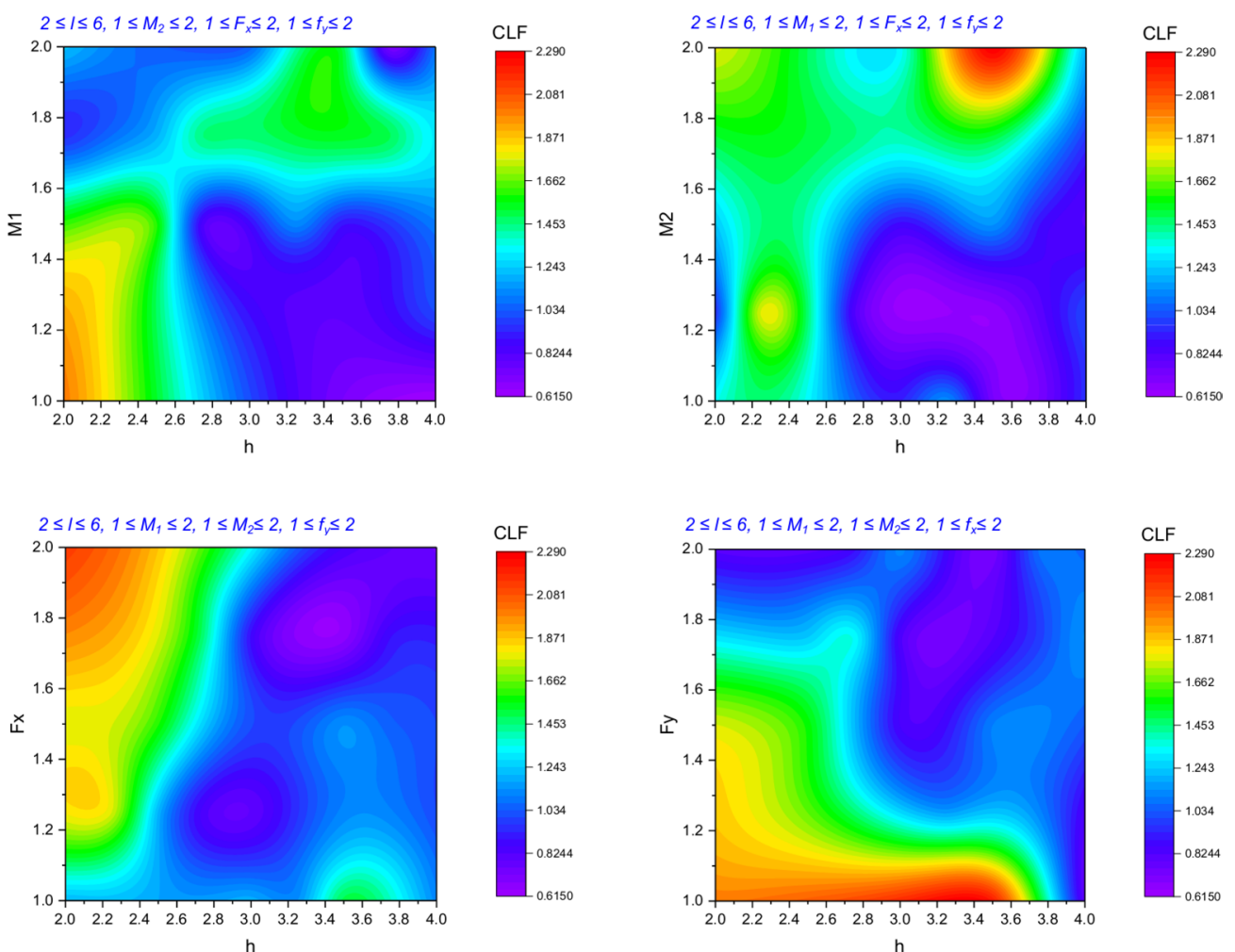

(b)
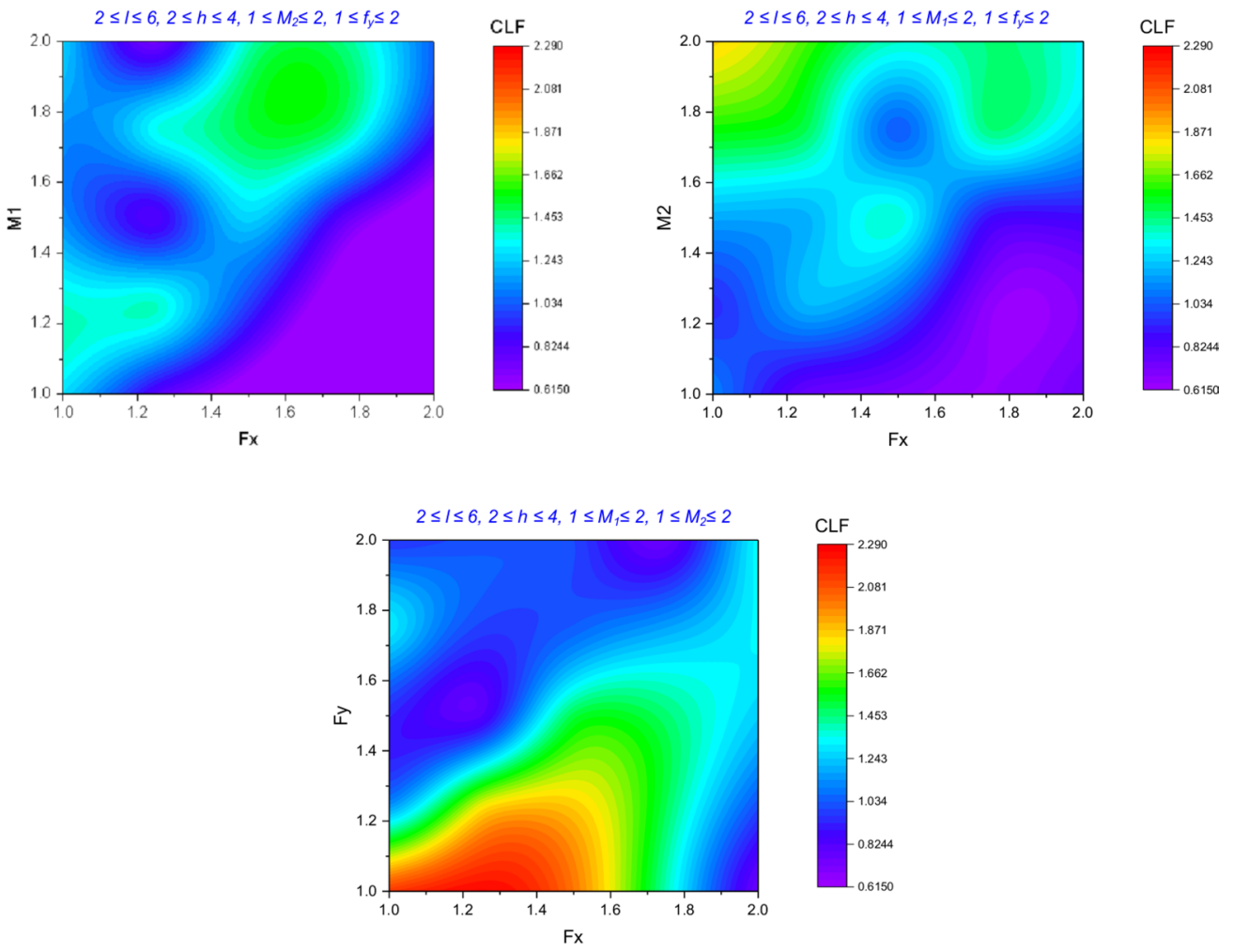

(c)

Fig. 9 (continued) 

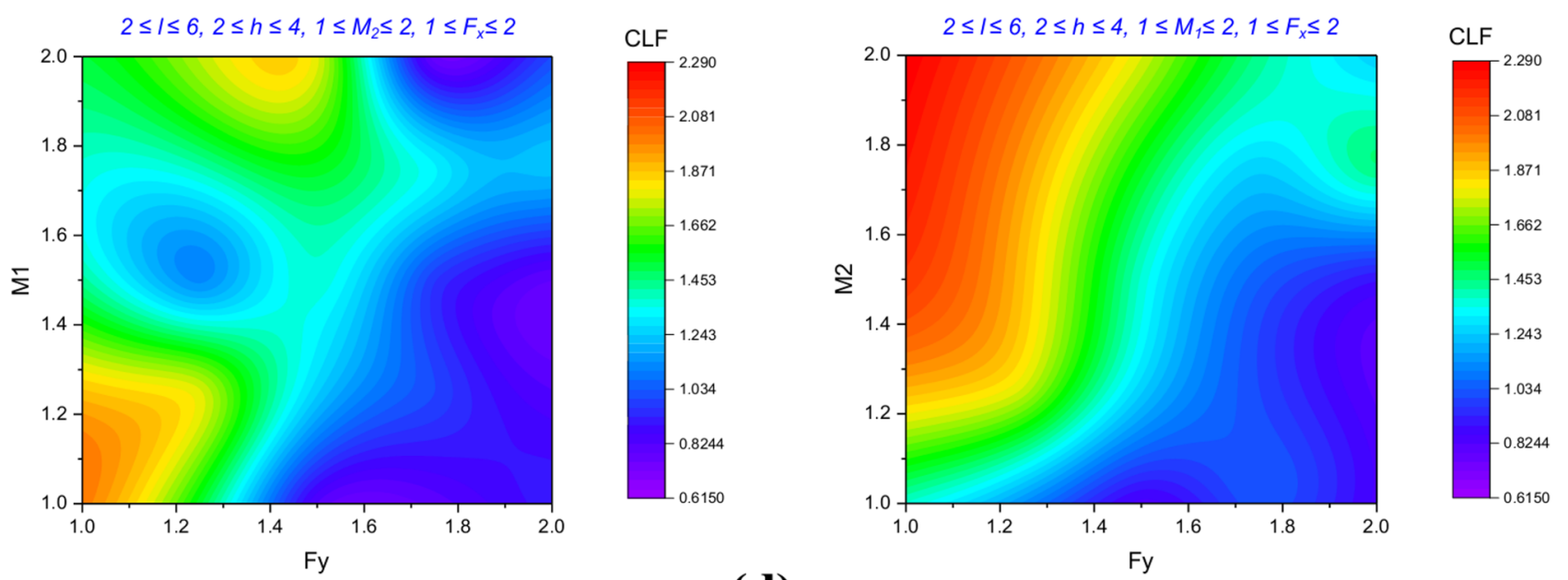

(d)

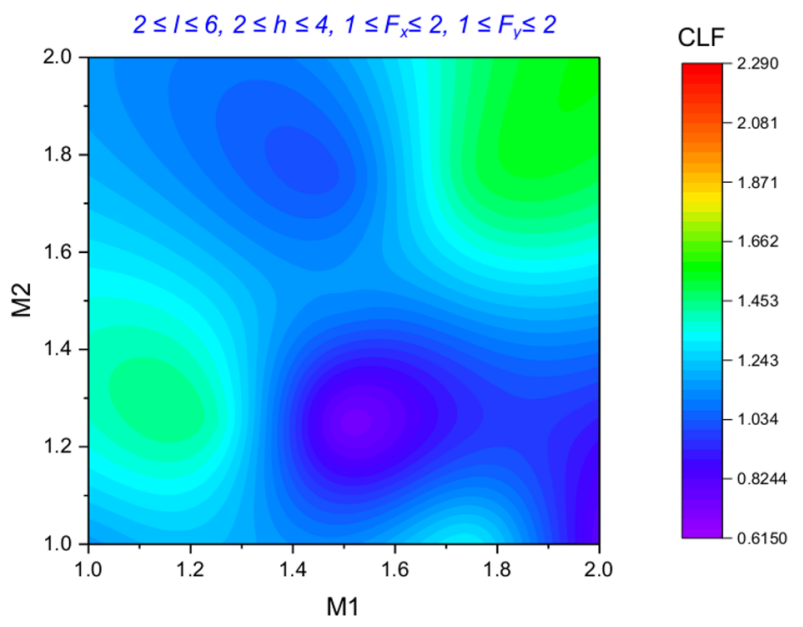

(e)

Fig. 9 (continued)

were considered as inputs and the collapse load factor (CLF) was considered as an output parameter. Afterward, the model was trained and tested. The values of statistical criteria used in training and testing processes showed that the obtained values of $R^{2}$ are more than 0.99 and the values of RMSE, MRE, and MAE are very close to zero for each considered example, which all are acceptable. Then, the related model functions are generated and the effects of each input parameters are studied. The results revealed that CLF has an inverse relationship with the value of height and length such that if the value of height is increasing the value of length must become lower and, conversely, if the value of length is enhancing the value of $h$ must have a reduction to have more CLF. Moreover, if some of the parameters of the plastic moment become higher, a turning point is made by increasing the values 

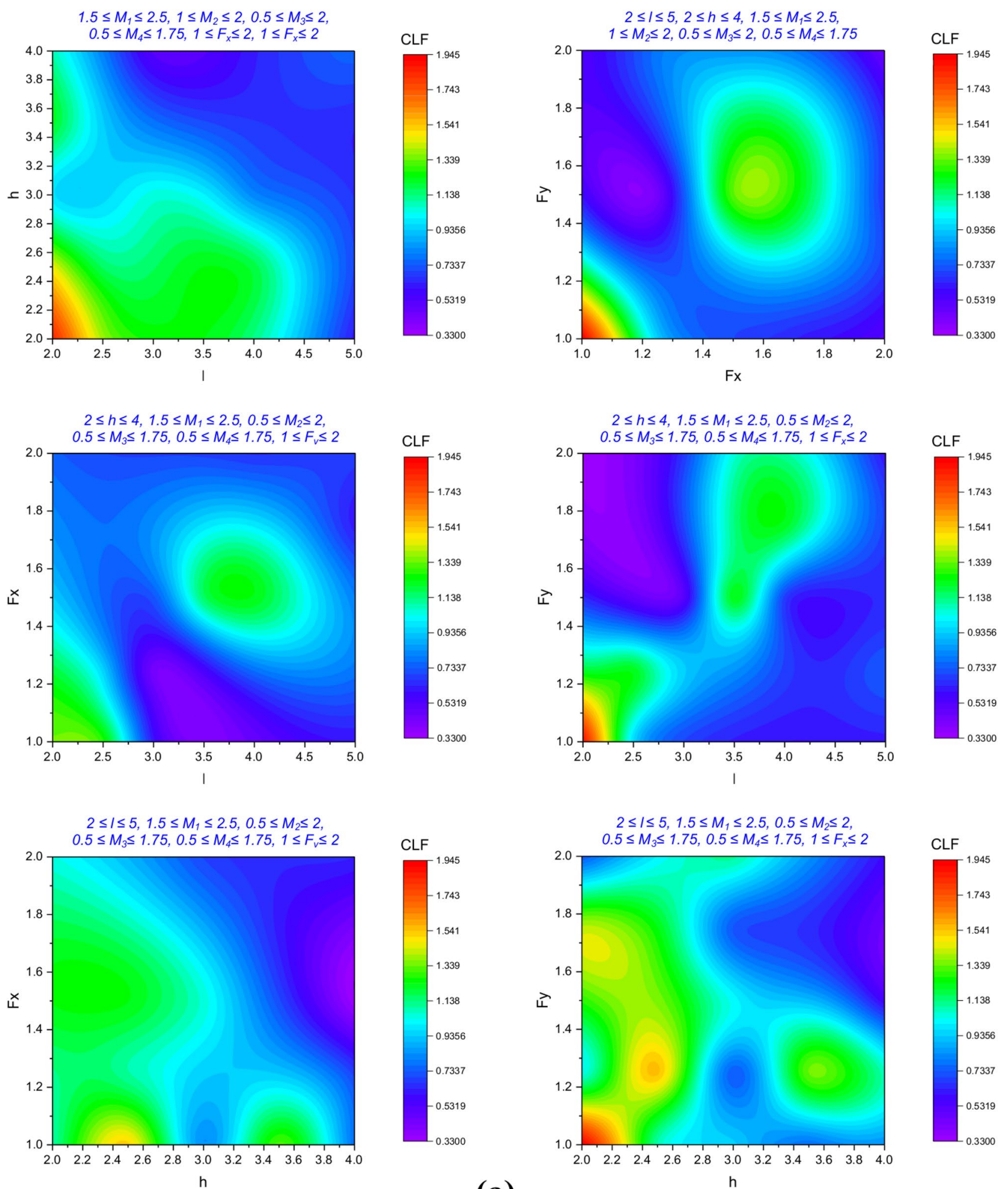

$2 \leq 1 \leq 5,1.5 \leq M_{1} \leq 2.5,0.5 \leq M_{2} \leq 2$,

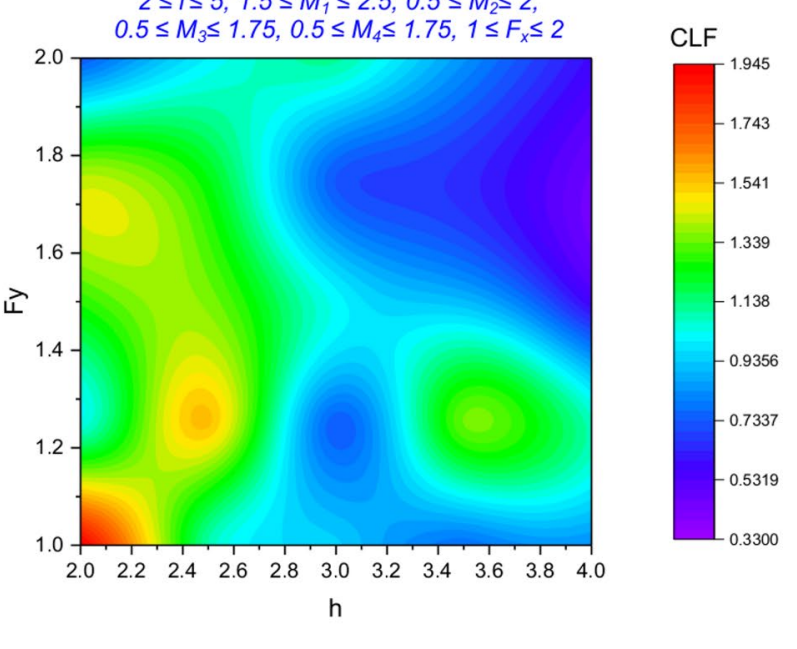

Fig. 10 Parametric analysis of input parameters including length of bay $l$, height of story $h$, and plastic moments $M$ influences on CLF obtained via generated model functions of the selected developed

network for example 2; effects of a length of bays, height of stories and applied forces, and $\mathbf{b}$ plastic moments and the other effective parameters all together on CLF 



(b)

Fig. 10 (continued) 

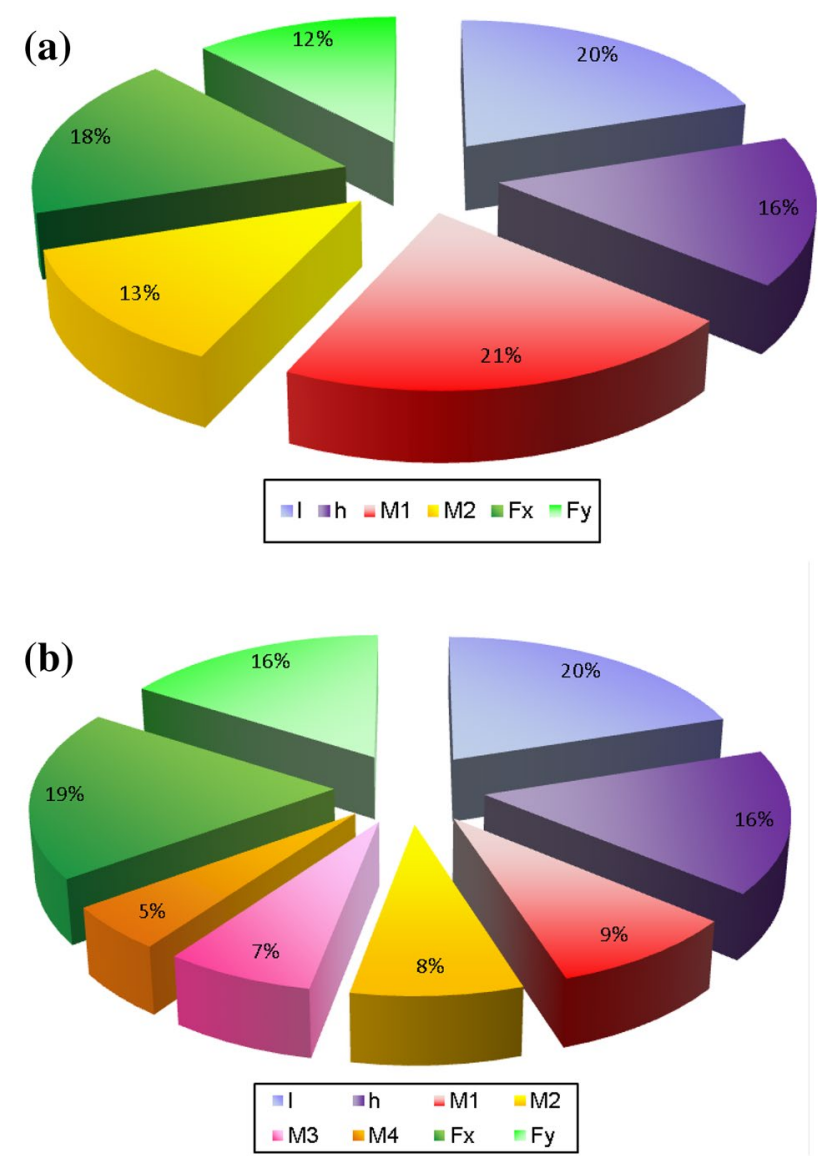

Fig. 11 Relative importance of each parameter on CLF for a example 1 and $\mathbf{b}$ example 2

Table 6 Ranking and the value of importance for each regarded inputs in both considered examples

\begin{tabular}{|c|c|c|c|c|}
\hline \multirow[t]{2}{*}{$\begin{array}{l}\text { Input } \\
\text { parameter }\end{array}$} & \multicolumn{2}{|c|}{$\begin{array}{l}\text { Example 1: one bay and } \\
\text { one story }\end{array}$} & \multicolumn{2}{|c|}{$\begin{array}{l}\text { Example 2: two bays } \\
\text { and two stories }\end{array}$} \\
\hline & $\begin{array}{l}\text { Importance } \\
(\%)\end{array}$ & Ranking & $\begin{array}{l}\text { Importance } \\
(\%)\end{array}$ & Ranking \\
\hline$L$ & 20 & 2 & 20 & 1 \\
\hline$H$ & 16 & 4 & 16 & 3 \\
\hline$M_{1}$ & 21 & 1 & 9 & 5 \\
\hline$M_{2}$ & 13 & 5 & 8 & 6 \\
\hline$M_{3}$ & - & - & 7 & 7 \\
\hline$M_{4}$ & - & - & 5 & 8 \\
\hline$F_{x}$ & 18 & 3 & 19 & 2 \\
\hline$F_{y}$ & 12 & 6 & 16 & 3 \\
\hline
\end{tabular}

of height and length. According to these results, when the networks are tuned finely and their parameters are adjusted carefully, satisfactory results can be obtained. As a result, ANN can be employed as an efficient tool plastic analysis and design of frames even the structures become more complex with a higher number of bays, stories, loads, and plastic moments.

Acknowledgements The authors want to thank Dr. Mohsen Jahanshahi, Assistant Professor of Civil Engineering Department at Sharif University of Technology- International Campus for his kind help to determine the exact values of CLP of each considered frames.

\section{Compliance with ethical standards}

Conflict of interest This work was accomplished in Sharif University of Technology, Iran where authors are employed and the authors stated that they have no conflict of interest.

Ethical statement Authors state that the research was conducted according to ethical standards.

\section{Appendix 1}

In the present study, only the effects of length of bays, height of stories and plastic moments on CLF are determined via a generated model function (see Fig. 12). 
(a)

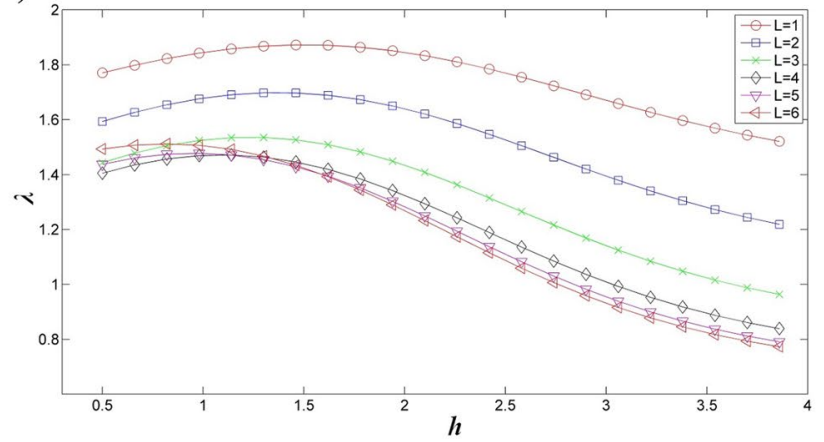

(c)

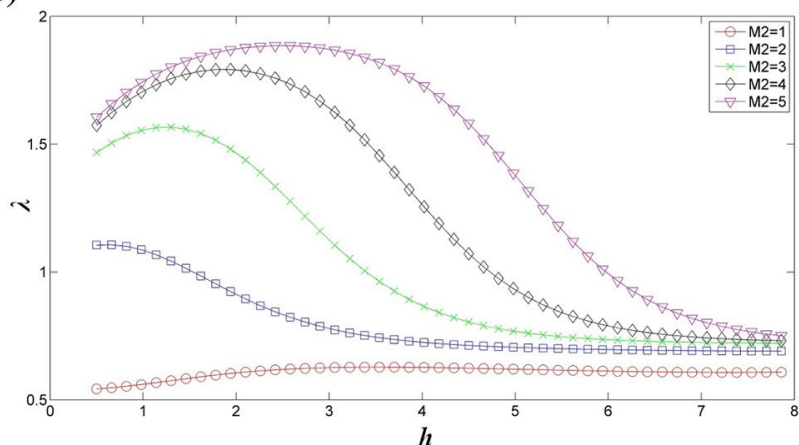

(e)

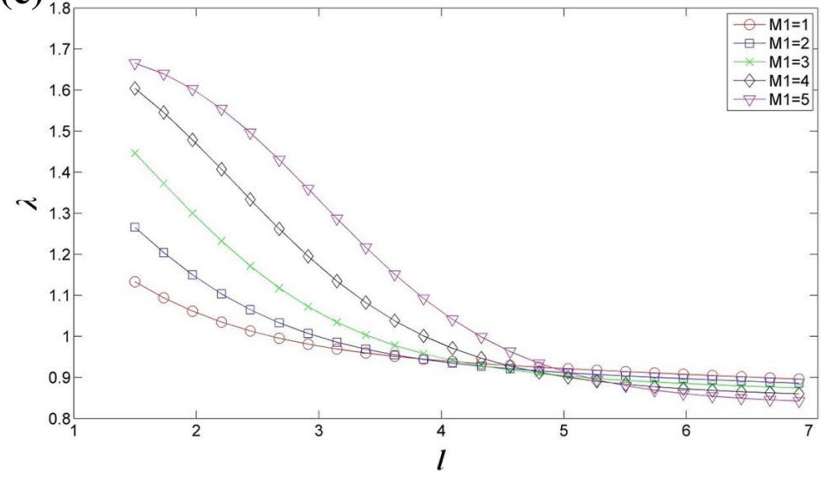

Fig. 12 Parametric analysis of input parameters including length of bay $l$, height of story $h$, and plastic moments $M$ influences on CLF obtained via generated model functions of the selected devel- (b)

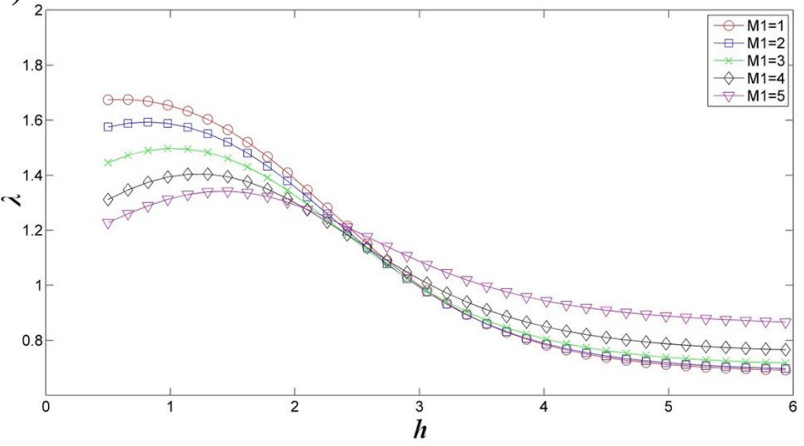

(d)

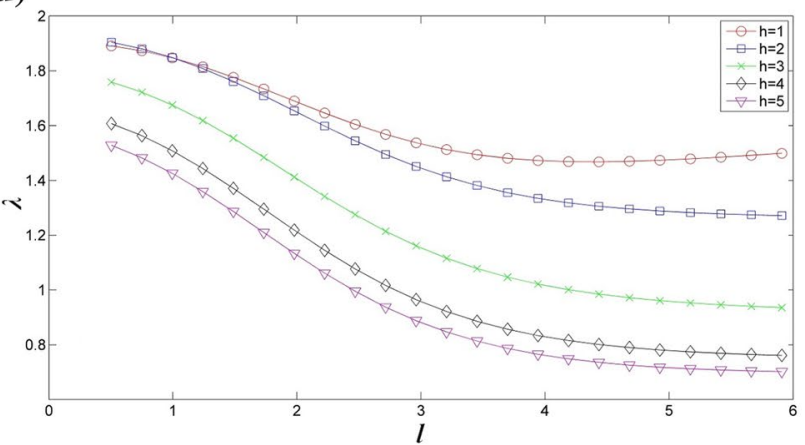

(f)

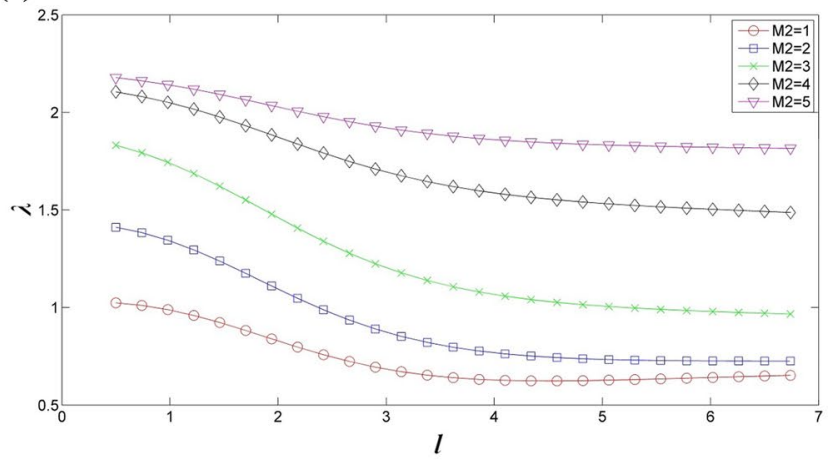

oped network for example 1: $\mathbf{a} \lambda=f(h, l), \mathbf{b} \lambda=f\left(h, M_{1}\right), \mathbf{c} \lambda=f\left(h, M_{2}\right), \mathbf{d}$ $\lambda=f(I, h), \mathbf{e} \lambda=f\left(l, M_{1}\right)$, and $\mathbf{f} \lambda=f\left(l, M_{2}\right)$ 


\section{Appendix 2}

\section{See Fig. 13.}

(a)

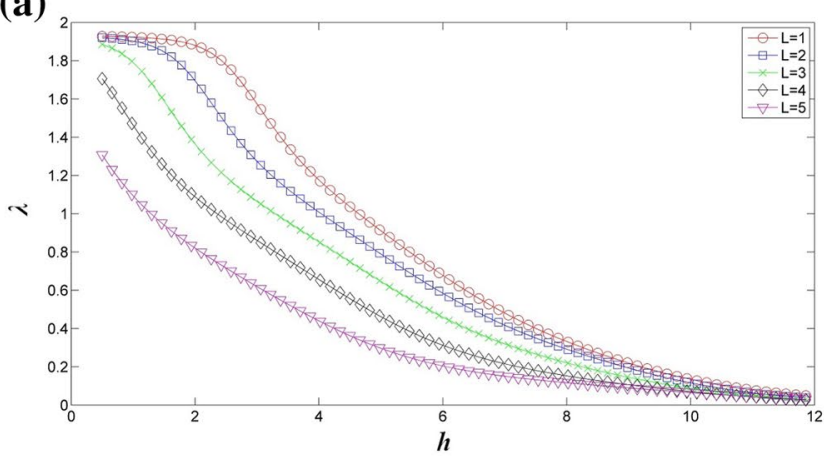

(c)

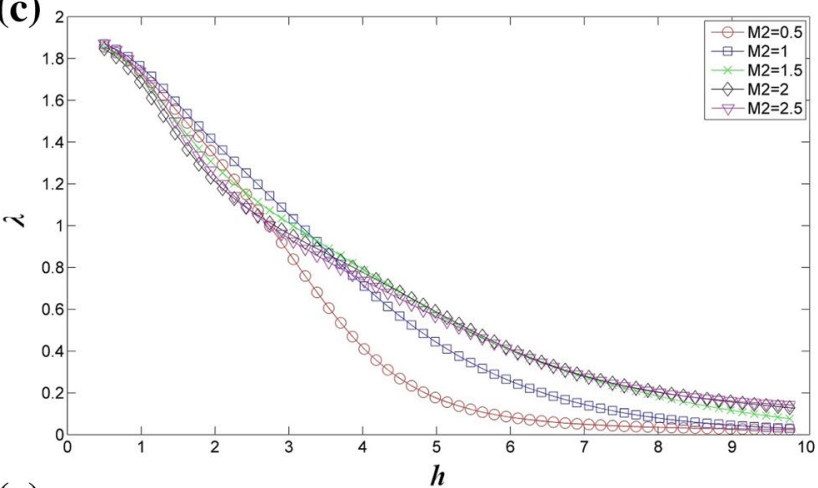

(e)

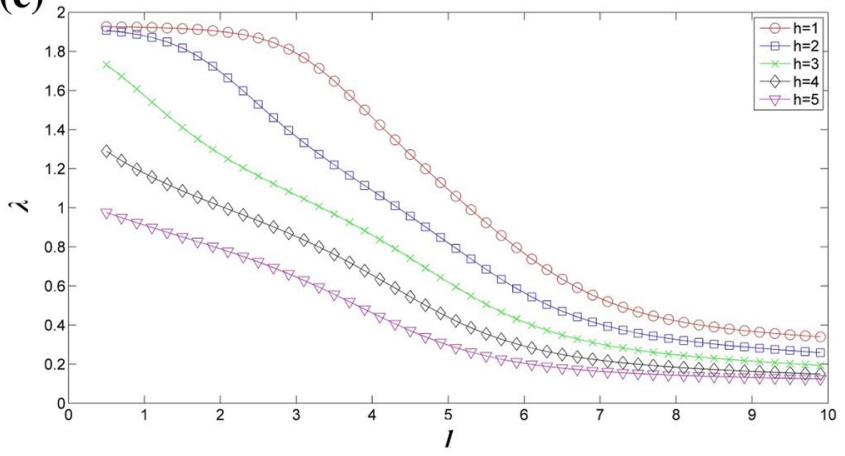

(g)

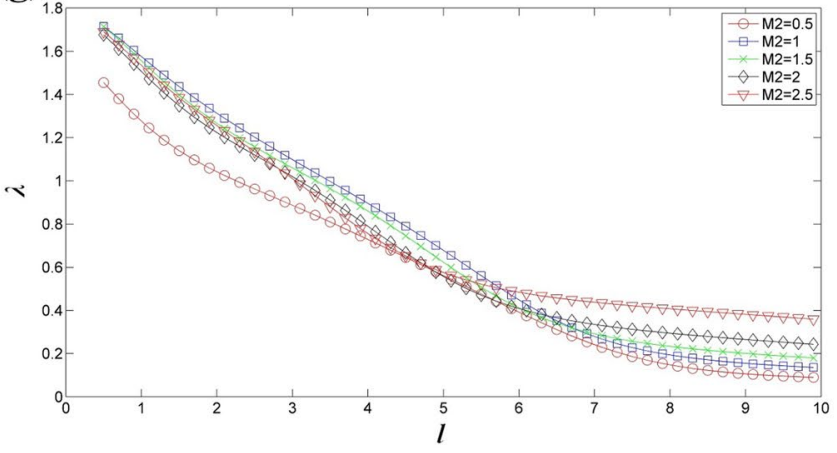

Fig. 13 Parametric analysis of input parameters including length of bay $l$, height of story $h$, and plastic moments $M$ influences on CLF obtained via generated model functions of the selected devel- (b)

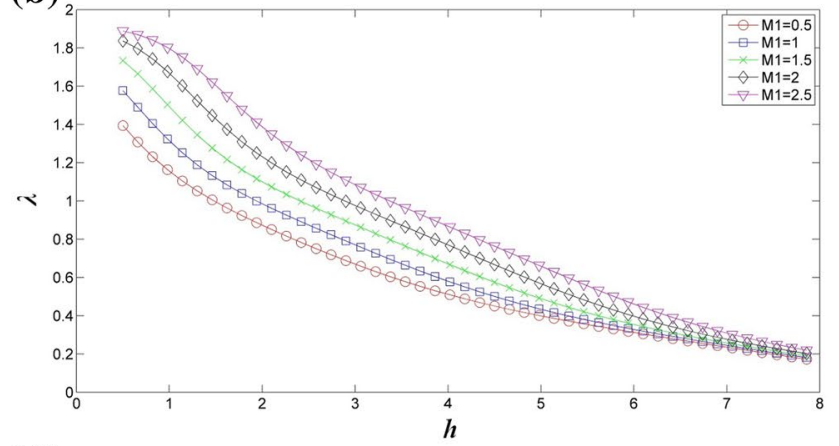

(d)

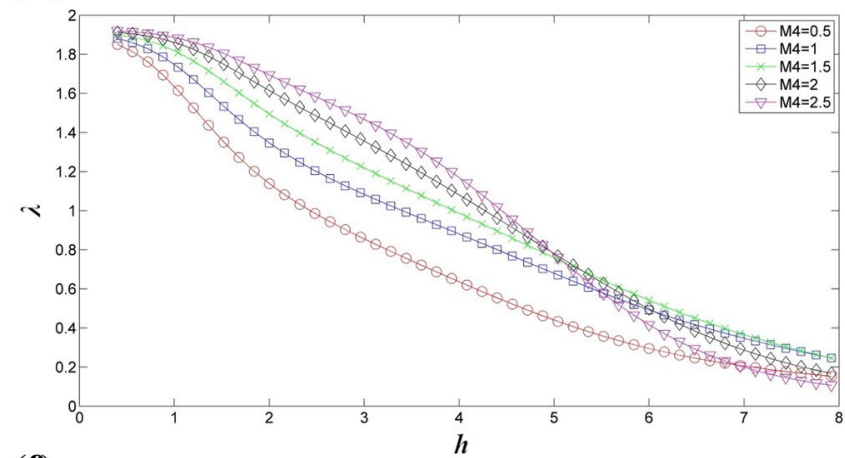

(f)

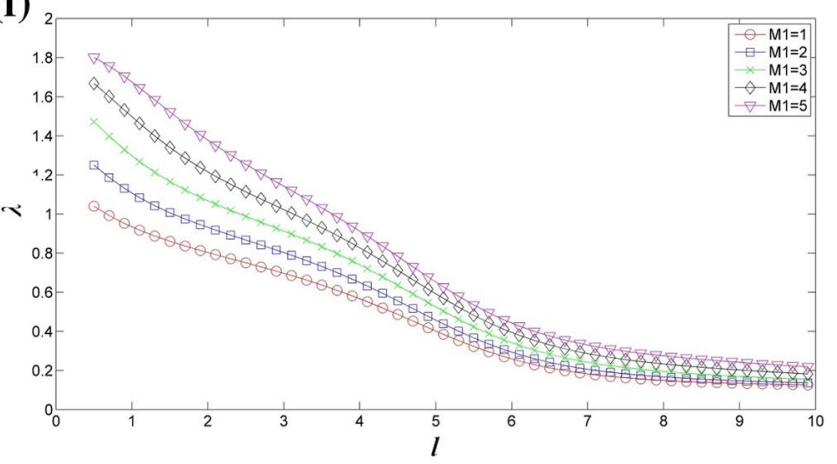

(h)



oped network for example 2: $\mathbf{a} \lambda=f(h, l), \mathbf{b} \lambda=f\left(h, M_{1}\right), \mathbf{c} \lambda=f\left(h, M_{2}\right), \mathbf{d}$ $\lambda=f\left(h, M_{4}\right), \mathbf{e} \lambda=f(l, h), \mathbf{f} \lambda=f\left(l, M_{1}\right), \mathbf{g} \lambda=f\left(l, M_{2}\right)$, and $\mathbf{h} \lambda=f\left(I, M_{4}\right)$

\section{SN Applied Sciences}




\section{References}

1. Neal BG, Symonds PS (1952) The rapid calculation of plastic collapse load for a framed structure. In: Proceedings of the institution of civil engineers, London, pp 58-71

2. Neal BG, Symonds PS (1952) The calculation of plastic loads for plane frames. In: International association for bridge and structural engineering, 4th congress, Cambridge and London

3. Neal BG, Symonds PS (1951) The calculations of collapse loads for framed structures. J Inst Civ Eng 35:21-40

4. Charnes A, Greenberg HJ (1959) Plastic collapse and linear programming. In: Summer meeting of the American mathematical society

5. Maier G, Pastor J, Ponter ARS, Weichert D (2003) Direct methods in limit and shakedown analysis. In: de Borst R, Mang HA (eds) Numerical and computational methods. Elsevier-Pergamon, Amsterdam

6. Maleki E, Sherafatnia K, Farrahi GH (2016) Size dependent energy release rate of notched FGM beams based on a modified couple stress theory. Mater Today Proc 3:2662-2671

7. Luongo Angelo, Contento Alessandro (2015) Nonlinear elastic analysis of steel planar frames under fire loads. Comput Struct 150:23-33

8. Mansouri I, Saffari H (2012) An efficient nonlinear analysis of 2D frames using a Newton-like technique. Arch Civ Mech Eng 12:485-492

9. Xiao N, Zhong H (2012) Non-linear quadrature element analysis of planar frames based geometrically exact beam theory. Int J Non-Linear Mech 47:481-488

10. Mahini MR, Moharrami H, Cocchetti G (2013) A dissipated energy maximization approach to elastic-perfectly plastic analy-sis of planar frames. Arch Mech 65:171-194

11. Nguyena PC, Kim SE (2017) Investigating effects of various base restraints on the nonlinear inelastic static and seismic responses of steel frames. Int J Non-linear Mech 89:151-167

12. Nguyen PC, Doan NTN, Ngo-Huu C, Kim SE (2014) Nonlinear inelastic response history analysis of steel frame structures using plastic-zone method. Thin-Walled Struct 85:220-233

13. Nguyen PC, Kim SE (2013) Nonlinear elastic dynamic analysis of space steel frames with semi-rigid connections. J Constr Steel Res 84:72-81

14. Nguyen PC, Kim SE (2014) An advanced analysis method for three-dimensional steel frames with semi-rigid connections. Finite Elem Anal Des 80:23-32

15. Nguyen PC, Kim SE (2014) Distributed plasticity approach for time-history analysis of steel frames including nonlinear connections. J Constr Steel Res 100:36-49

16. Nguyen PC, Kim SE (2014) Nonlinear inelastic time-history analysis of three-dimensional semi-rigid steel frames. J Constr Steel Res 101:192-206

17. Nguyen PC, Kim SE (2015) Second-order spread-of-plasticity approach for nonlinear time-history analysis of space semi-rigid steel frames. Finite Elem Anal Des 105:1-15

18. Nguyen PC, Kim SE (2016) Advanced analysis for planar steel frames with semi-rigid connections using plastic-zone method. Steel Compos Struct 21:1121-1144

19. Nguyen PC, Kim SE (2017) Investigating effects of various base restraints on the nonlinear inelastic static and seismic responses of steel frames. Int J Non-linear Mech 89:151-167

20. Greco A, Cannizzaro F, Pluchino A (2017) Seismic collapse prediction of frame structures by means of genetic algorithms. Eng Struct 143:152-168

21. Aminian P, Javid MR, Asghari A, Gandomi AH, Arab Esmaeili M (2011) A robust predictive model for base shear of steel frame structures using a hybrid genetic programming and simulated annealing method. Neural Comput Appl 20:1321-1332

22. Pinto Lacides R, Zambrano Alejandro R (2014) Unsupervised neural network approach to frame analysis of conventional buildings. Int J Commun Network Syst Sci 7:203-211

23. Greco A, Cannizzaro F, Pluchino A (2019) Automatic evaluation of plastic collapse conditions for planar frames with vertical irregularities. Eng Comput 35(1):57-73

24. Greco A, Cannizzaro F, Pluchino A (2019) An improved ant colony optimization algorithm and its applications to limit analysis of frame structures. Eng Optim 143:152-168

25. Kaveh A, Jahanshahi M (2008) Plastic limit analysis of frames using ant colony systems. Comput Struct 86:1152-1163

26. Cao M, Qiao P, Ren Q (2009) Improved hybrid wavelet neural network methodology for time-varying behavior prediction of engineering structures. Neural Comput Appl 18:821-832

27. Aydin K, Kisi O (2012) Damage detection in Timoshenko beam structures by multilayer perceptron and radial basis function networks. Neural Comput Appl 24:583-597

28. Yahya S, Moghavvemi M, Mohamed Haider AF (2014) Artificial neural networks aided solution to the problem of geometrically bounded singularities and joint limits prevention of a three dimensional planar redundant manipulator. Neurocomputing 137:34-46

29. Jiang $X$ et al (2016) Fuzzy stochastic neural network model for structural system identification. Mech Syst Signal Process. https ://doi.org/10.1016/j.ymssp.2016.05.030

30. Jahanshahi M, Maleki E, Ghiami A (2016) On the efficiency of artificial neural networks for plastic analysis of planar frames in comparison with genetic algorithms and ant colony systems. Neural Comput Appl. https://doi.org/10.1007/s0052 1-016-2228-5

31. Deeks AJ (1996) Automatic computation of plastic collapse loads for frames. Comput Struct 60:91-102

32. Maleki N, Kashanian S, Maleki E, Nazari M (2017) A novel enzyme based biosensor for catechol detection in water samples using artificial neural network. Biochem Eng J 128:1-11

33. Maleki N, Maleki E (2015) Modeling of cathode Pt/C electrocatalyst degradation and performance of a PEMFC using artificial neural network. In: Proceedings of the the international conference on engineering \& MIS 2015 (ICEMIS '15), Istanbul, Turkey, pp 1-8. https://doi.org/10.1145/2832987.2833000

34. Zopf C, Kaliske M (2017) Numerical characterisation of uncured elastomers by a neural network based approach. Comput Struct 182:504-525

35. Reza Kashyzadeh K, Maleki E (2017) Experimental investigation and artificial neural network modeling of warm galvanization and hardened chromium coatings thickness effects on fatigue life of AISI 1045 carbon steel. Fail Anal Prev 17:1276-1287

36. Facchini L, Betti M, Biagini P (2014) Neural network based modal identification of structural systems through output-only measurement. Comput Struct 138:183-194

37. Maleki E, Maleki N (2016) artificial neural network modeling of $\mathrm{pt} / \mathrm{c}$ cathode degradation in PEM fuel cell. J Electron Mater 45:3822-3834

38. Maleki E, Farrahi GH (2018) Modeling of conventional and severe shot peening influence on properties of high carbon steel via artificial neural network. IJE Trans B: Appl 31:382-393

39. Maleki E, Zabihollah A (2016) Modeling of shot peening effects on the surface properties of $(\mathrm{TiB}+\mathrm{TiC}) / \mathrm{Ti}-6 \mathrm{Al}-4 \mathrm{~V}$ composite employing artificial neural networks. Materiali In Tehnologije 50(6):43-52

40. Maleki E, Reza Kashyzadeh K (2017) Effects of the hardened nickel coating on the fatigue behavior of CK45 steel: experimental, finite element method, and artificial neural network modeling. Iran J Mater Sci Eng 14:81-99 
41. Maleki E (2016) Modeling of severe shot peening effects to obtain nanocrystalline surface on cast iron using artificial neural network. Mater Today Proc 3:2197-2206

42. Zhao J, Ding $H$, Zhao W, Huang M, Wei D, Jiang Z (2014) Modelling of the hot deformation behaviour of a titanium alloy using constitutive equations and artificial neural network. Comput Mater Sci 92:47-56

43. Maleki E, Unal O, RezaKashyzadeh K (2018) Fatigue behavior prediction and analysis of shot peened mild carbon steels. Int J Fatigue 116:48-67

44. Maleki E, Sherafatnia K (2016) Investigation of single and dual step shot peening effects on mechanical and metallurgical properties of $18 \mathrm{CrNiMo7-6}$ steel using artificial neural network. Int J Mater Mech Manuf 4:100-105

45. Elangovan K, Narayanan CS, Narayanasamy R (2010) Modelling of forming limit diagram of perforated commercial pure aluminium sheets using artificial neural network. Comput Mater Sci 47:1072-1078

46. Maleki E (2015) Artificial neural networks application for modeling of friction stir welding effects on mechanical properties of 7075-T6 aluminum alloy. IOP Conf Ser Mater Sci Eng 103:012034. https://doi.org/10.1088/1757-899X/103/1/012034

47. Maleki E, Farrahi GH, Sherafatnia K (2016) Application of artificial neural network to predict the effects of severe shot peening on properties of low carbon steel. In: Öchsner A, Altenbach $\mathrm{H}$ (eds) Mach Join Modif Adv Mater. Springer, Singapore, pp 45-60

Publisher's Note Springer Nature remains neutral with regard to jurisdictional claims in published maps and institutional affiliations. 